\title{
Boundary-to-bulk maps for AdS causal wedges and the Reeh-Schlieder property in holography
}

\author{
Ian A. Morrison \\ Department of Physics, McGill University, \\ Montreal, QC H3A 2T8, Canada \\ E-mail: imorrison@physics.mcgill.ca
}

\begin{abstract}
In order to better understand how AdS holography works for sub-regions, we formulate a holographic version of the Reeh-Schlieder theorem for the simple case of an AdS Klein-Gordon field. This theorem asserts that the set of states constructed by acting on a suitable vacuum state with boundary observables contained within any subset of the boundary is dense in the Hilbert space of the bulk theory. To prove this theorem we need two ingredients which are themselves of interest. First, we prove a purely bulk version of Reeh-Schlieder theorem for an AdS Klein-Gordon field. This theorem relies on the analyticity properties of certain vacuum states. Our second ingredient is a boundaryto-bulk map for local observables on an AdS causal wedge. This mapping is achieved by simple integral kernels which construct bulk observables from convolutions with boundary operators. Our analysis improves on previous constructions of AdS boundary-to-bulk maps in that it is formulated entirely in Lorentz signature without the need for large analytic continuation of spatial coordinates. Both our Reeh-Schlieder theorem and boundary-tobulk maps may be applied to globally well-defined states constructed from the usual AdS vacuum as well more singular states such as the local vacuum of an AdS causal wedge which is singular on the horizon.
\end{abstract}

KEYwORDS: Gauge-gravity correspondence, AdS-CFT Correspondence

ARXIV EPRINT: 1403.3426 


\section{Contents}

1 Introduction 1

2 The bulk Reeh-Schlieder theorem $\quad 4$

2.1 Bulk theory basics 4

2.2 Locally analytic states and the RS theorem 5

3 Boundary-to-bulk maps $\quad 9$

$\begin{array}{llr}3.1 & \text { Boundary theory basics } & 9\end{array}$

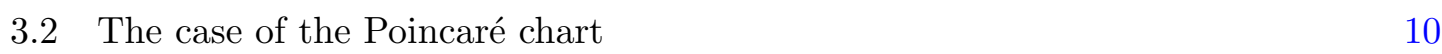

$\begin{array}{lll}3.3 & \text { AdS causal wedges } & 12\end{array}$

$\begin{array}{ll}3.4 & \text { The case of the AdS-Rindler wedge } \\ \end{array}$

$\begin{array}{lll}4 & \text { Discussion } & 16\end{array}$

$\begin{array}{ll}\text { A Wave front sets } & 19\end{array}$

B An example transformation to an AdS causal wedge 20

C WKB approximations of AdS-Rindler Klein-Gordon modes 22

\section{Introduction}

One of the most striking features of quantum physics is the phenomena of quantum entanglement. A familiar example of entanglement in quantum mechanics is provided by EPR pairs. In local quantum field theory (LQFT) there is a sense in which entanglement is stronger, or at least more ubiquitous, and this is embodied in the Reeh-Schlieder theorem $[1,2]$. Consider an LQFT on a manifold $\mathcal{M}$ and consider the set of local observables $\mathcal{A}(\mathcal{O})$ whose support is contained in a sub-region $\mathcal{O} \subset \mathcal{M}$. The Reeh-Schlieder theorem states that the set of states generated by acting on a suitable vacuum state with members of $\mathcal{A}(\mathcal{O})$ is dense upon the Hilbert space. In essence, in LQFT observables always have long-range correlations which, even if small, can be exploited; an observer with limited spacetime but unlimited resources can explore the entire Hilbert space.

It is natural to ask how this strong notion of quantum entanglement fits into the AdS/CFT correspondence [3, 4], or more generally gauge/gravity duality [5]. In general AdS/CFT is a correspondence between two quantum theories, a $d$-dimensional boundary conformal field theory (CFT), and $d+1$-dimensional bulk quantum gravity theory. When quantum gravity is sufficiently weak the bulk theory becomes local and the duality becomes one between two LQFTs. In this setting one expects the following formulation of the ReehSchlieder theorem: the set of CFT states constructed by acting on a suitable CFT vacuum 


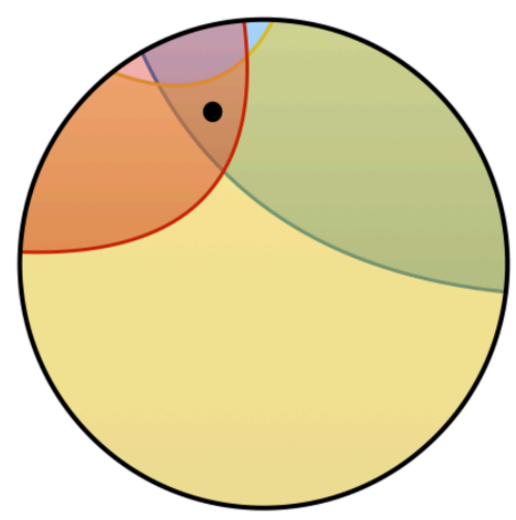

Figure 1. An an equal-time surface of global AdS. A bulk observable is at the black dot. The yellow, red, and blue shaded regions denote AdS causal wedges which include the support of the observable. The boundaries of these wedges have no mutual intersection. The observable may be computed from CFT correlation functions on the boundary of any one of these wedges.

with observables supported in any sub-region of the boundary provides a dense set of states for the bulk Hilbert space.

In this paper we prove a holographic form of the Reeh-Schlieder theorem in the simple case of a Klein-Gordon scalar field on a fixed AdS background. This requires two ingredients, each of which is of independent interest. Our first ingredient is a purely bulk formulation of the Reeh-Schlieder theorem. This theorem applies to states built atop suitably analytic vacuum states. Examples of such states include the usual AdS vacuum as well as the natural "local vacua" of AdS sub-regions such as the AdS-Rindler vacuum. In order to make the theorem holographic we define a suitable algebra of local boundary observables $\mathcal{A}_{\text {bndry }}$, along with the natural algebra of local bulk observables $\mathcal{A}_{\text {bulk }}$. We then consider the theory restricted to a sub-region $\mathcal{W}$ of AdS known as an AdS causal wedge. The conformal boundary of an AdS causal wedge is the causal development $\mathcal{D}$ of a compact, spherical region on an equal time hypersurface of the boundary. Our second ingredient is the construction of a 1-to-1 map for the restricted algebras of observables: $\mathcal{A}_{\text {bndry }}(\mathcal{D}) \mapsto \mathcal{A}_{\text {bulk }}(\mathcal{W}) .{ }^{1}$ Thus any state constructed by applying bulk observables supported within $\mathcal{W}$ to a vacuum state may be equivalently constructed by applying boundary observables supported within $\mathcal{D}$. The causal wedge has a length scale (the radius of $\mathcal{D}$ ) which we may make as small as we like. In this way we prove the holographic Reeh-Schlieder theorem.

Aspects of our results have been anticipated in many ways. Perhaps the most direct antecedents are discussions of the gravity duals of CFT density matrices [6, 7], and in particular of the gravity duals of Rindler CFTs [8-11]. Also relevant is the perspective gained from gravity computations of CFT entanglement entropy [12-15] and related quantities (see e.g., [16-23]). These investigations provide significant evidence to suggest that given $\mathrm{CFT}$ data on $\mathcal{D}$ the largest bulk region from which we expect to recover "fine-grained" information about the bulk theory is the associated causal wedge $\mathcal{W}$. Our boundary-to-

\footnotetext{
${ }^{1}$ Strictly speaking the boundary algebra $\mathcal{A}_{\text {bndry }}(\mathcal{D})$ is larger than the bulk algebra $\mathcal{A}_{\text {bulk }}(\mathcal{W})$ so the former contains a representation of the latter.
} 
bulk map shows that, at least for this simple theory, this "fine-grained" information is in fact the entire bulk observable algebra $\mathcal{A}_{\text {bulk }}(\mathcal{W})$. It is widely believed that data on $\mathcal{D}$ provides access to "coarse-grained" information about the bulk theory beyond $\mathcal{W}$ (see e.g., [24-27]). Our work shows that in this model the course-grained information accessible is precisely that implied by the Reeh-Schlieder theorem: using elements of $\mathcal{A}_{\text {bndry }}(\mathcal{D})$ one may well-approximate, but not distinguish, any bulk state.

We now describe our process and results in more detail. In the first part of our study we prove a version of the Reeh-Schlieder (RS) theorem for Klein-Gordon fields quantized in the Poincare chart of AdS (the extension to global AdS is straightforward). As is always the case for the RS theorem, this theorem applies to states constructed from a suitably analytic vacuum state. Our theorem holds for a class of "locally analytic" vacuum states which are Gaussian states whose Wightman functions satisfy a certain analytic wave front set condition. Roughly speaking, these vacuum states are as analytic as possible within their domain of local analyticity. The key property enjoyed by these states is a unique notion of analytic continuation; from this property the RS theorem readily follows. Since our definition of a locally analytic state is local a state can be locally analytic when restricted to a sub-region of AdS but fail beyond. The usual AdS vacuum is an example of a state which is locally analytic on all of the Poincaré chart. Examples of states which are locally analytic only within sub-regions of AdS include the natural AdS-Rindler vacuum and more generally the local vacuum of any AdS causal wedge. These are the natural zero temperature vacuum states defined relative to the timelike Killing vector field of the sub-region. Thus, our RS theorem applies not only to the familiar basis of Poincaré particle states, but to finite-energy excitations of these local vacua as well. Essentially any state consistent with the semi-classical approximation (i.e. a state with well-defined, finite averaged stress-energy tensor) is included in our treatment.

In the second part of our study we construct a boundary-to-bulk map for local observables on an AdS causal wedge. ${ }^{2}$ Similar boundary-to-bulk maps for Klein-Gordon fields have been discussed before, notably in the very nice papers [28-31] — for generalizations see [32-36]. While these works represent an excellent initial effort, there are some technical improvements we need in order to construct a successful map for a causal wedge. These works employ an integral kernel $K(X \mid y)$ which creates a bulk operator $\Phi(X)$ from it's boundary value $\phi(y)$ :

$$
\Phi(X)=\int d^{d} y \sqrt{-\gamma(y)} K(X \mid y) \phi(y) .
$$

Here $X$ is a $d+1$-dimensional bulk coordinate, $y$ is a $d$-dimensional boundary coordinate, and $\gamma(y)$ is the induced metric on the conformal boundary. When considering sub-regions of AdS the integral kernel $K(X \mid y)$ is a distribution - in a convenient Fourier representation the kernel can diverge exponentially at large momenta - and thus it's convolution with correlation functions which are themselves distributions requires careful analysis. Previous authors dealt with these divergences by performing a large analytic continuation in the

\footnotetext{
${ }^{2}$ The reader should confuse this map with the bulk-to-boundary propagator. Our map is a map from the boundary value of the normalizable bulk field to it's bulk profile. There are no boundary sources and no non-normalizable part of the bulk field.
} 
spatial coordinates of $y$ - a much larger foray into the complex plane than is implemented by an $i \epsilon$ prescription - and this has led some to question whether these boundary-to-bulk maps are actually well-defined in the physically correct Lorentz signature [11, 37]. ${ }^{3}$ The more refined analysis we present here shows that despite the distributional nature of the $K(X \mid y)$ these boundary-to-bulk maps converge on states relevant for our RS theorem.

We use the following line of reasoning to show that the boundary-to-bulk map for AdS causal wedges converges. The distributional character of CFT correlation functions is constrained by basic aspects of the theory - ingredients such as positivity and the non-negativity of the energy spectrum. From these considerations we may determine the largest class of boundary "test functions" which have well-defined convolution with CFT correlators. This class includes certain distributions in addition to functions. Our main task is to show that the test functions constructed using the integral kernel $K(X \mid y)$ appropriate to an AdS causal wedge are in fact members of this class. Obviously, this analysis is a bit technical, but it is made rather simple by the use of a tool known as the $C^{\infty}$ wave front set of a distribution. This object characterizes the singularities of a distribution in an invariant manner. Lest these tools dismay the reader, we also show through explicit calculation that the boundary-to-bulk map converges on both the usual CFT vacuum as well as the local vacuum for a boundary sub-region $\mathcal{D}$.

This paper proceeds quite simply. In section 2 we set up and prove the bulk RS theorem. In section 3 we establish the boundary-to-bulk maps for AdS causal wedges. We close with a discussion in section 4. Some mathematical background and lengthy calculations are collected in the appendices.

\section{The bulk Reeh-Schlieder theorem}

\subsection{Bulk theory basics}

This section serves to establish our conventions for the bulk Klein-Gordon theory. For simplicity we focus on quantization in the Poincaré chart of $D=d+1$-dimensional AdS (PAdS). The line element is

$$
d s^{2}=\frac{\ell^{2}}{Z^{2}}\left(-d T^{2}+d Z^{2}+d \vec{X}^{2}\right), \quad Z \in(0,+\infty),
$$

where $\ell$ is the AdS radius and $Z$ is the AdS "radial" direction such that $Z=0$ corresponds to the conformal boundary. The extension of our analysis to global AdS is straightforward.

We consider a real scalar field $\Phi(X)$ obeying the Klein-Gordon equation

$$
\left(\square-M^{2}\right) \Phi(X)=0 .
$$

AdS has a timelike conformal boundary the thus is not globally hyperbolic, so we must impose boundary conditions in order to have well-posed Cauchy evolution. As is well

\footnotetext{
${ }^{3}$ Indeed, the analysis of [11] and particularly [37] shows that in general for less-symmetric, asymptoticallyAdS spaces the integral kernel $K(X \mid y)$ is highly distributional. Thus in genereal one cannot side-step the issue via analytic continuations.
} 
known, solutions to the equation of motion (2.2) have two behaviors as $Z \rightarrow 0$ :

$$
\Phi(X) \sim Z^{\Delta}+Z^{d-\Delta},
$$

where $\Delta=\frac{d}{2}+\sqrt{\frac{d^{2}}{4}+M^{2} \ell^{2}}$ such that $M^{2} \ell^{2}=\Delta(\Delta-d)$. We impose the standard boundary condition that $\Phi(X)=O\left(Z^{\Delta}\right)$ as $Z \rightarrow 0$. This boundary condition is invariant under AdS isometries and is sufficient to guarantee unique Cauchy evolution [38-40].

Upon quantization the scalar field becomes an operator and must be evaluated within a correlation function $\langle\ldots\rangle_{\Psi}$ of some quantum state $\Psi$. Correlation functions satisfy the equation of motion

$$
\left(\square-M^{2}\right)\langle A \Phi(X) B\rangle_{\Psi}=0,
$$

where $A, B$ are arbitrary operator insertions, as well as the canonical commutation relations

$$
\left\langle A\left[\Phi\left(X_{1}\right), \Phi\left(X_{2}\right)\right] B\right\rangle_{\Psi}=i \Delta\left(X_{1}, X_{2}\right)\langle A B\rangle_{\Psi},
$$

where $\Delta\left(X_{1}, X_{2}\right)$ is the commutator function, a.k.a. the advanced-minus-retarded fundamental solution [41], which is unique given our choice of boundary conditions. We take as the basic observables of the theory the "smeared" operators

$$
\Phi[F]:=\int d^{D} X \sqrt{-g(X)} F(X) \Phi(X), \quad F \in C^{\infty}(\mathrm{PAdS}) .
$$

We define the bulk algebra of local observables $\mathcal{A}_{\text {bulk }}$ to be the unital *-algebra generated by finite sums of finite products of the basic elements (2.6). The restriction of this algebra to a sub-region $\mathcal{O} \subset$ PAdS is denoted $\mathcal{A}_{\text {bulk }}(\mathcal{O})$. Quantum states are positive linear functionals on this algebra.

Technically the algebra $\mathcal{A}_{\text {bulk }}$ does not include observables constructed from composite operators such as $\Phi^{2}(X)$ or the all-important stress tensor. Obviously we must have these observables in order to completely describe bulk physics. In particular, we regard a finite averaged stress-energy tensor to be an additional criteria for a state to be deemed physically reasonable (otherwise the semi-classical approximation is invalid). In this work we consider states for which composite operators may be constructed via normal ordering. Thus for these states one may construct directly from $\mathcal{A}_{\text {bulk }}$ a larger "Wick polynomial algebra" which includes observables constructed from composite operators, though we need not present these details here - see, e.g., [42-45].

\subsection{Locally analytic states and the RS theorem}

For typical field theories on AdS there exists a class of states which are highly analytic; this can be traced back to the fact that AdS is a complex analytic manifold. While a mathematician might call these states sparse in the same way that analytic functions are sparse on the set of smooth functions, for most physical questions this set of analytic states provide the vacuum states of interest. In this section we define a set of locally analytic states which, roughly speaking, are as analytic as the usual AdS vacuum. This analyticity property is local so a state may be locally analytic in a sub-region of AdS and fail to be so 
elsewhere. Locally analytic states enjoy a notion of unique analytic continuation. This is the property we need to formulate a Reeh-Schlieder theorem.

For simplicity we restrict attention to quasi-free (a.k.a. Gaussian) states. The analyticity properties of a quasi-free state are simply those of it's Wightman function. A Wightman function is a distribution, and the analyticity of distributions is considerably more nuanced than that of functions. The analyticity properties of a distribution are nicely encoded in a mathematical tool known as the analytic wave front set $\left(\mathrm{WF}_{\mathrm{A}}\right)$ of a distribution. This is a rather technical object, so for the moment we provide a colloquial description of wave front sets that will likely be sufficient for a first reading. We provide a precise definition of the wave front set, along with further introduction, in appendix A. Let us first describe the $C^{\infty}$ wave front set (WF) which we will use later in section 3 . This object provides a precise characterization of the singularities of a distribution by listing, for each point in position space at which the distribution is singular, the directions in a locally-constructed Fourier space for which the function fails behave as a smooth function. The wave front set is defined locally and transforms covariantly under general diffeomorphisms; it is naturally thought of as a subset of the cotangent bundle of the manifold. One may similarly define the analytic wave front set which describes the locations and momentum directions in which a distribution fails to be analytic — for details see appendix A.

Consider the familiar global AdS vacuum $\Omega$. The Wightman function of $\Omega$ is $[4,46]$

$$
\begin{aligned}
W_{\Omega}\left(X_{1}, X_{2}\right): & =\left\langle\Phi\left(X_{1}\right) \Phi\left(X_{2}\right)\right\rangle_{\Omega} \\
& =\frac{\ell^{(2-D)} \Gamma(\Delta) \xi_{12}^{\Delta}}{2^{\Delta+1} \pi^{d / 2} \Gamma\left(\Delta-\frac{d}{2}+1\right)} 2 F_{1}\left[\frac{\Delta}{2}, \frac{\Delta+1}{2} ; \Delta-\frac{d}{2}+1 ; \xi_{12}^{2}\right] .
\end{aligned}
$$

Here ${ }_{2} F_{1}$ is the Gauss hypergeometric function, $\xi_{12}=\left(1+u_{12}\right)^{-1}+i \epsilon s_{12}, u_{12}$ is the $S O(d, 2)$-invariant chordal distance between $X_{1}$ and $X_{2},{ }^{4}$ and $s_{12}=+(-) 1$ for $X_{1}$ in the causal future (past) of $X_{2}$ and equal to 0 else. This Wightman function is analytic away from $\xi_{12}=+(-) 1$ corresponding to null (antipodal) separation. More precise information is given by it's analytic wave front set:

$$
\mathrm{WF}_{\mathrm{A}}\left(W_{\Omega}\right)=\left\{\left(x_{1}, k_{1} ; x_{2}, k_{2}\right) \in\left(T^{*}(\mathrm{PAdS})\right)^{2} \backslash\{0\} \mid \xi_{12}= \pm 1, k_{1} \in V^{+}, k_{1} \sim-k_{2}\right\} .
$$

Following notation employed in appendix A, $T^{*}$ denotes the tangent space, $\{0\}$ the zero section, $V^{+}$the closed forward lightcone, ${ }^{5}$ and $k_{1} \sim k_{2}$ denotes an equality after parallel transporting $k_{1}$ to $x_{2}$. Essentially (2.9) states that the singularities of $W_{\Omega}$ are of locally positive frequency, much like the coincident singularity of the Poincaré-invariant vacuum of Minkowski QFT.

One could also consider states which have the same analyticity properties of the AdS vacuum in a sub-region of PAdS but fail to be analytic everywhere. Examples of such

\footnotetext{
${ }^{4}$ In Poincaré coordinates $(2.1)$

$$
\xi_{12}=\frac{2 Z_{1} Z_{2}}{Z_{1}^{2}+Z_{2}^{2}+\left(x_{1}-x_{2}\right)^{2}},
$$

where we use the notation $X=(Z, x)$.

${ }^{5}$ The closed forward (backward) cones are defined to be $V^{+(-)}:=\left\{k \in \mathbb{R}^{d-1,1} \mid k^{2} \leq 0,+(-) k^{0}>0\right\}$. We use Lorentz signature for Fourier space and the tangent space.
} 

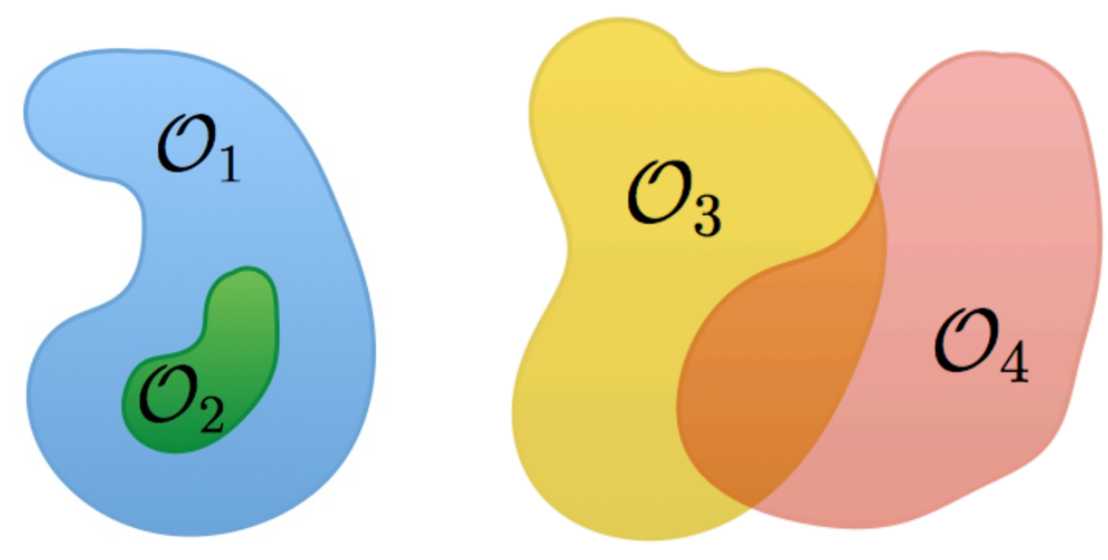

Figure 2. Depictions of the domains of analyticity referenced in the text.

states include the "local vacua" of AdS causal wedges and the AdS-Rindler wedge. The Wightman function of such a state is as analytic as $\Omega$ within it's defining sub-region, but has additional singularities on the boundary horizon as well as beyond the region. With these states in mind we define:

Definition 2.1 A locally analytic state $\Psi$ on $\mathcal{A}_{\text {bulk }}(\mathcal{O})$ is a quasi-free state whose Wightman function $W_{\Psi}\left(X_{1}, X_{2}\right)$ satisfies the analytic wave front set condition

$$
\mathrm{WF}_{\mathrm{A}}\left(W_{\Psi}\right) \subseteq\left\{\left(x_{1}, k_{1} ; x_{2}, k_{2}\right) \in\left(T^{*}(\mathcal{O})\right)^{2} \backslash\{0\} \mid \xi_{12}= \pm 1, k_{1} \in V^{+}, k_{1} \sim-k_{2}\right\} .
$$

The AdS vacuum $\Omega$ is locally analytic on all of PAdS; we will verify later that natural vacuum of an AdS causal wedge is locally analytic on the wedge.

Locally analytic states enjoy a notion of unique analytic continuation. This follows from a very general form of the "edge of the wedge theorem" [47] familiar from Minkowski space QFT reformulated here in terms of analytic wave front sets:

Theorem 2.2 (Edge of the wedge theorem (Proposition 5.3 of [48])) Let $\mathcal{M}$

be a real analytic connected manifold and $u$ a distribution (in the distribution space dual to that of smooth functions) with the property that

$$
\mathrm{WF}_{\mathrm{A}}(u) \cap-\mathrm{WF}_{\mathrm{A}}(u)=\emptyset .
$$

Then for each non-void open subset $\mathcal{O} \subset \mathcal{M}$ if the restriction of $u$ to $\mathcal{O}$ vanishes then $u=0$.

The following simple lemmas follow immediately:

Lemma 2.3 Let $\Psi$ be locally analytic on $\mathcal{O}_{1}$. If a $2 n$-pt function of $\Psi$ vanishes when restricted to an open set $\mathcal{O}_{2} \subset \mathcal{O}_{1}$ then $\Psi=0$ on $\mathcal{O}_{1}$.

Proof. The state $\Psi$ is quasi-free so if a 2n-pt function vanishes on $\mathcal{O}_{2}$ it implies $W_{\Psi}=0$ on $\mathcal{O}_{2}$. $W_{\Psi}$ satisfies the criteria $(2.11)$ so if it vanishes on $\mathcal{O}_{2}$ it vanishes on $\mathcal{O}_{1}$. Thus $\Psi=0$ on $\mathcal{O}_{1}$. 
Lemma 2.4 Let $\Psi_{3 / 4}$ be locally analytic on $\mathcal{O}_{3 / 4}$, and let $\mathcal{O}_{3} \cap \mathcal{O}_{4}$ be non-void. If $W_{\Psi_{3}}$ $W_{\Psi_{4}}=0$ on an open set of $\mathcal{O}_{3} \cap \mathcal{O}_{4}$ then $\Psi_{3}=\Psi_{4}$ on $\mathcal{O}_{3} \cap \mathcal{O}_{4}$ and $\Psi_{3 / 4}$ provides the unique locally analytic extension of $\Psi_{4 / 3}$ on $\mathcal{O}_{3 / 4}$.

Proof. This follows immediately from the fact that $W_{\Psi_{3 / 4}}$ each satisfy (2.11).

These lemmas show how the locally analytic extension of a state is uniquely defined, but they do not prove the existence of such an extension. Indeed, as the example of AdS causal wedge vacua show, in general there is no such extension.

Let us turn the discussion to the more general class of states constructed by acting on a locally analytic state with members of $\mathcal{A}_{\text {bulk }}$. It is easy to show that such states are no more singular than locally analytic states, but are not necessarily as analytic. The following lemma follows immediately from the properties of wave front sets:

Lemma 2.5 Let $\Psi$ be locally analytic on $\mathcal{O}_{1}$ and let $\mathcal{O}_{2} \subset \mathcal{O}_{1}$ be a sub-region such that it's causal complement $\overline{\mathcal{O}}_{2}$ on $\mathcal{O}_{1}$ is non-void. Then the state $\Psi_{2}=A_{2} \Psi$ with $A_{2} \in \mathcal{A}_{\text {bulk }}\left(\mathcal{O}_{2}\right)$ is locally analytic on at least $\overline{\mathcal{O}}_{2}$.

Despite being less analytic, states generated from locally analytic states enjoy a weaker property reminiscent of analyticity which is embodied in the Reeh-Schlieder theorem:

Theorem 2.6 (Reeh-Schlieder theorem) Let $\Psi$ be locally analytic on $\mathcal{O}_{1}$ and let $\mathcal{O}_{2} \subset$ $\mathcal{O}_{1}$. The linear spans of the sets

$$
S_{1}=\left\{A_{1} \Psi \mid A_{1} \in \mathcal{A}_{\text {bulk }}\left(\mathcal{O}_{1}\right)\right\}, \quad S_{2}=\left\{A_{2} \Psi \mid A_{2} \in \mathcal{A}_{\text {bulk }}\left(\mathcal{O}_{2}\right)\right\}
$$

are equal.

Proof. Let $\Psi_{1} \in S_{1}$ and let $C=\Phi\left(X_{1}\right) \ldots \Phi\left(X_{2 n}\right)$ be any finite string of operators with arguments restricted to $\mathcal{O}_{2}$. Consider

$$
F=\left\langle\Psi_{1}|C| \Psi\right\rangle=\left\langle A_{1}^{*} C\right\rangle_{\Psi} .
$$

If $F$ is non-zero for all $A_{1} \in \mathcal{A}_{\text {bulk }}\left(\mathcal{O}_{1}\right)$ then the assertion of the theorem holds. If $F=0$ for some $A_{1}$ then it follows from lemma 2.3 that $\Psi=0$ on $\mathcal{O}_{1}$ and the theorem holds.

To understand the implications of this theorem consider when $\Psi$ is the AdS vacuum $\Omega$ and $\mathcal{O}_{1}$ is PAdS. Then the set $S_{1}$ is the set of finite-energy excitations of $\Omega$, and $S_{2}$ is the set of states which may be constructed using observables contained in $\mathcal{O}_{2}$. The RS theorem states that there is no state in $S_{1}$ orthogonal to the set $S_{2}$. If we consider as the Hilbert space of the bulk theory the usual one for which $\Omega$ is cyclic then it follows that the set $S_{2}$ is dense on this Hilbert space. Thus any state in this Hilbert space may be approximated with arbitrary precision by a state on $S_{2}$. In other words, by judicious application of operators in $\mathcal{O}_{2}$ to $\Omega$ one may construct a global state which well-approximates any finite-energy state everywhere on PAdS. An important corollary is:

Theorem 2.7 Consider the same configuration as theorem 2.6, and in addition let $\overline{\mathcal{O}}_{2}$ be the non-void causal complement of $\mathcal{O}_{2}$ on $\mathcal{O}_{1}$. If $A_{2} \in \mathcal{A}_{\text {bulk }}\left(\mathcal{O}_{2}\right)$ annihilates any state in $S_{1}$ then it annihilates all states in $S_{1}$. 
Proof. From theorem 2.6 it follows that the set $\bar{S}_{2}=\left\{B_{2} \Psi \mid B_{2} \in \mathcal{A}_{\text {bulk }}\left(\overline{\mathcal{O}}_{2}\right)\right\}$ spans $S_{1}$. All such $B_{2}$ commute with $A_{2}$. Thus if $A_{2} \Psi=0$ then $A_{2} B_{2} \Psi=0$ and so $A_{2}$ annihilates $S_{1}$. The conclusion is unchanged if we exchange $\Psi$ for any member $A_{1} \Psi \in S_{1}$.

This corollary makes precise the difference between a dense set of states and the entire Hilbert space. If we think again of the case where $\Psi=\Omega$ and $\mathcal{O}_{1}=$ PAdS then the theorem states that an observer confined to $\mathcal{O}_{2}$ cannot construct a set of exact annihilation operators for the span $S_{1}$ and thus the $\mathcal{O}_{2}$ observer cannot exactly determine the quantum state. For further interpretation of the RS theorem and related corollaries see, e.g., [2, 47].

The interpretations we have just given can also be applied to cases where $\mathcal{O}_{1}$ is a sub-region of PAdS and $\Psi$ is any locally analytic state on $\mathcal{O}_{1}$.

\section{Boundary-to-bulk maps}

In this section we change gears and describe the boundary-to-bulk map for local observables on an AdS causal wedge. After establishing some technical details in section 3.1 we review the construction of such a map for the Poincaré chart in section 3.2. We introduce AdS causal wedges in section 3.3. Finally in section 3.4 we examine in detail the boundary-tobulk map for the AdS-Rindler wedge.

\subsection{Boundary theory basics}

In this section we review the construction of the boundary theory from the bulk theory. We know this is familiar territory, but we will need some rather fine details for our later analysis so we might as well state everything clearly now.

The conformal boundary of the $d+1$-dimensional Poincaré chart is Minkowski space $\mathbb{R}^{d-1,1}$. Following the standard AdS/CFT prescription we define the boundary operator $\phi(x)$ via the limit

$$
\phi(x):=\lim _{Z \rightarrow 0} Z^{\Delta} \Phi(X) .
$$

We use the notation $X=(Z, x)$ with $x$ a $d$-dimensional coordinate. AdS transformations in the bulk act as conformal transformations on the conformal boundary, and under such actions $\phi(x)$ transforms as a conformal field of weight $\Delta$. Thus $\phi(x)$ constructed in this way may be used to define a CFT on $d$-dimensional Minkowski space. This CFT what we refer to as "the CFT" or "the boundary theory."

We say that every bulk state $\Psi$ induces a boundary state $\psi$; so for instance, the AdSinvariant bulk state $\Omega$ induces a conformally-invariant boundary state $\omega$. Obviously, the boundary states induced by quasi-free states are also quasi-free. We restrict attention to boundary states induced by bulk states constructed from locally analytic states within their domain of analyticity. The 2-pt functions of such boundary states have reasonable singularity structure. In fact, they satisfy the so-called "microlocal spectrum condition" [45]. For the case at hand this condition states that the 2-pt function $W_{\psi}\left(x_{1}, x_{2}\right)$ of a state $\psi$ has a $C^{\infty}$ wave front set contained in

$$
\mathrm{WF}\left(W_{\psi}\right) \subseteq\left\{\left(x_{1}, k_{1} ; x_{2}, k_{2}\right) \in\left(T^{*} \mathbb{R}^{d-1,1}\right)^{2} \backslash\{0\}|| x_{1}-\left.x_{2}\right|^{2}=0, k_{1} \in V^{+}, k_{1} \sim-k_{2}\right\} .
$$


In plain words this says that $W_{\psi}\left(x_{1}, x_{2}\right)$ is singular at most when $x_{1}$ and $x_{2}$ are nullseparated, and that the singularities are of locally-positive frequency. One may regard (3.2) as a very basic statement about the OPE structure of the theory.

For our purposes we define the algebra of local boundary observables $\mathcal{A}_{\text {bndry }}$ to be the unital $*$-algebra composed of finite sums of finite products of the smeared boundary field

$$
\phi[f]=\int d^{d} x \sqrt{-\gamma(x)} f(x) \phi(x), \quad f \in \mathscr{T},
$$

where $\gamma(-x)$ is the determinant of the induced metric on the boundary and $\mathscr{T}$ is a suitable class of test functions. In textbook introductions to Minkowski QFT the class of test functions is usually taken to be smooth functions, and there are many good physical and mathematical reasons for this choice $[2,47]$. For the purposes of holography it turns out that we need a different, strictly larger class of test functions whose precise definition is somewhat technical. The issue here is that we desire an algebra of boundary observables which is large enough to contain compactly-supported representations of bulk observables (this will be made more clear in later sections.) This requires that we consider as "test functions" not only smooth functions but also certain distributions. Since correlation functions of $\phi(x)$ are themselves distributions, the observables (3.3) involve the point-wise product of distributions and care must be taken to assure that these objects are well-defined.

As described in appendix A, wave front sets allow us to provide a precise criteria for when the point-wise product of two distributions is unique. Provided that the boundary theory 2-pt functions satisfy (3.2), the largest class of "test functions" $\mathscr{T}$ which yield welldefined convolutions $\phi[f]$ is the set of distributions on $\mathbb{R}^{d-1,1}$ whose members $f$ satisfy the wave front set condition

$$
\mathrm{WF}(f) \subseteq\left\{(x, k) \in T^{*} \mathbb{R}^{d-1,1} \backslash\{0\} \mid k^{2}>0\right\} .
$$

In plain words this says that $f$ is a distribution whose local Fourier transform behaves like that of a smooth function in timelike and null momentum directions. Thus the convolution $\phi[f]$ contains no overlapping singular directions and is unambiguous.

\subsection{The case of the Poincaré chart}

We now review the construction of the boundary-to-bulk map for local observables on Poincaré AdS following closely [28-31] — for some complementary remarks on this process see [49]. Our goal is to compute local bulk observables in a bulk state $\Psi$ given only it's boundary counterpart $\psi$. In this section we assume we have access to the 2-pt function of $\psi$ everywhere on the Minkowski boundary, i.e. we have ${ }^{6}$

$$
\left\langle\phi\left(x_{1}\right) \phi\left(x_{2}\right)\right\rangle_{\psi}=\int \frac{d^{d} k_{1}}{(2 \pi)^{d}} \frac{d^{d} k_{2}}{(2 \pi)^{d}} \theta\left(-k_{1}^{2}\right) \theta\left(-k_{2}^{2}\right) e^{i k_{1} \cdot x_{1}} e^{-i k_{2} \cdot x_{2}} \widehat{W}_{\psi}\left(k_{1}, k_{2}\right) .
$$

\footnotetext{
${ }^{6}$ States defined on the entire $\mathbb{R}^{d-1,1}$ boundary have Wightman functions whose support in momentum space is limited to $\left|k^{0}\right| \geq|\vec{k}|$. This follows from the fact that spectrum of the boundary translation operator $P_{\mu}$ is contained in the closed forward lightcone $V^{+}$. This is mimicked in the Poincaré bulk where the restriction $\left|k^{0}\right| \geq|\vec{k}|$ also follows from the non-negativity of the energy spectrum.
} 
A very intuitive way to proceed is as follows. Recall that in the bulk there exists a complete set of mode solutions to the Klein-Gordon equation. We may take these modes to have the form $e^{i k \cdot x} V_{k}(Z)$, where the function $V_{k}(Z)$ which contains the $Z$-dependence and may be taken to be real and satisfy $V_{k}=Z^{\Delta}\left(1+O\left(Z^{2}\right)\right)$ as $Z \rightarrow 0$. This asymptotic behavior is as prescribed by our boundary conditions; the normalization is a convenient choice. Using these bulk Klein-Gordon mode functions we may extend the 2-pt function of $\psi$ into the bulk simply by "dressing" each Fourier mode in (3.5) with the appropriate $V_{k}(Z)$ :

$$
\left\langle\Phi\left(x_{1}\right) \Phi\left(x_{2}\right)\right\rangle_{\Psi}=\int \frac{d^{d} k_{1}}{(2 \pi)^{d}} \frac{d^{d} k_{2}}{(2 \pi)^{d}} \theta\left(-k_{1}^{2}\right) \theta\left(-k_{2}^{2}\right) e^{i k_{1} \cdot x_{1}} e^{-i k_{2} \cdot x_{2}} \widehat{W}_{\psi}\left(k_{1}, k_{2}\right) V_{k_{1}}\left(z_{1}\right) V_{k_{2}}\left(z_{2}\right) .
$$

By construction this bulk 2-pt function satisfies the equation of motion and limits to the boundary 2-pt function of $\psi$. If the boundary 2-pt function is positive then so too is the bulk 2-pt function. Because we assume that $\psi$ satisfies the microlocal spectrum condition (3.2), it follows that the momentum integrals in (3.6) converge for spacelike-separated bulk points; for points with timelike or null separation further analysis is required.

The process of computing a bulk observation in $\Psi$ may be regarded within the boundary theory as an observation of $\psi$. This perspective has some technical advantages. In this way of looking at things the goal is to show that for each element $\Phi[F] \in \mathcal{A}_{\text {bulk }}$ there exists a representative element $\phi\left[f_{F}\right] \in \mathcal{A}_{\text {bndry }}$. Taken together the set $\left\{\phi\left[f_{F}\right]\right\} \subset \mathcal{A}_{\text {bndry }}$ forms a representation of the bulk algebra of observables. Alternatively, we can say that there exists a 1-to- 1 map $\mathcal{A}_{\text {bndry }} \mapsto \mathcal{A}_{\text {bulk }}$ which effectively constructs a bulk state from it's boundary value: $\psi \mapsto \Psi$. This map is provided by the integral kernel $^{7}$ [29-31]

$$
K(X \mid y)=\int \frac{d^{d} k}{(2 \pi)^{d}} \theta\left(-k^{2}\right) e^{i k \cdot(x-y)} V_{k}(Z) .
$$

This object takes one bulk argument $X$ and one boundary argument $y$. For each bulk test function $F(X)$ we associate a boundary test function $f_{F}(y)$ via

$$
f_{F}(y):=\int d^{D} X \sqrt{-g(X)} F(X) K(X \mid y), \quad F \in C^{\infty}(\operatorname{PAdS}) .
$$

By construction the kernel satisfies the bulk equation of motion with respect to $X$ and is also consistent with the boundary conditions imposed on the bulk theory. Note that the kernel is a distribution and is not smooth. For fixed $Z$ the bulk Klein-Gordon mode functions $V_{k}(Z)$ decay like an inverse power of $|k|$ as $|k| \rightarrow \infty$. What is important, however, is not the behavior of $K(X \mid y)$ but that of $f_{F}(y)$. Because $F(X)$ is smooth a smooth function of $X$ it follows that the $f_{F}(y)$ defined in (3.8) is a smooth function of $y$, so indeed $f_{F} \in \mathscr{T}$. The test functions $f_{F}(y)$ which extract bulk observables from the boundary theory are essentially as nice as can be and any globally well-defined Minkowski CFT state yields finite correlators for the set $\left\{\phi\left[f_{F}\right]\right\}$.

\footnotetext{
${ }^{7}$ As has been discussed by previous authors, the representation of the kernel $K(X \mid y)$ is not unique. This is because the CFT correlation functions on which it acts do not have support for spacelike momenta, so one is free to add to $K(X \mid y)$ any object whose Fourier transform is supported only at spacelike momenta. Of course the map $\mathcal{A}_{\text {bndry }} \mapsto \mathcal{A}_{\text {bulk }}$ is unaltered by such additions.
} 

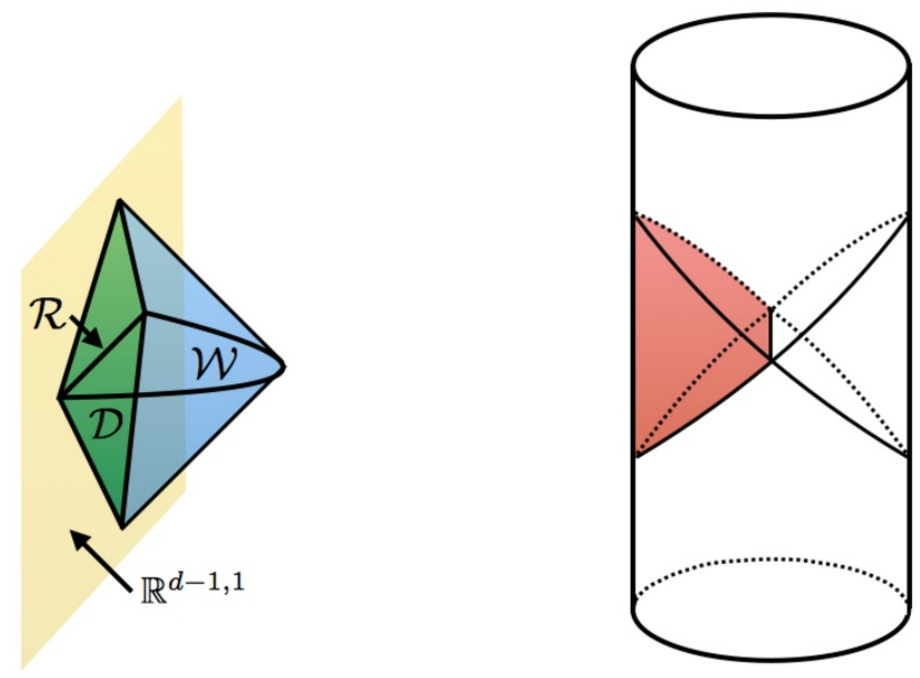

Figure 3. Left: an AdS causal wedge. Right: global AdS with the AdS-Rindler wedge highlighted.

\subsection{AdS causal wedges}

Next we would like to construct a boundary-to-bulk map for a sub-region of AdS which is smaller than the Poincaré chart and likewise employs a smaller region of the boundary than the entire Minkowski space. As has been discussed by many before (see e.g., $[6,7,23]$ ) the natural sub-region to consider is the AdS causal wedge. Consider a spherical region of an equal-time hypersurface on $\mathbb{R}^{d-1,1}$ :

$$
\mathcal{R}=\left\{x \in \mathbb{R}^{d-1,1} \mid x^{0}=\bar{x}^{0}, \sum_{i=1}^{d-1}\left(x^{i}-\bar{x}^{i}\right)^{2}=L\right\} .
$$

This region has origin $\bar{x}$ and radius $L$. Let $D_{\text {bndry }}^{+(-)}$denote the future (past) domain of dependence on $\mathbb{R}^{d-1,1}$. Then we refer the causal domain of dependence of $\mathcal{R}$ as a causal development $\mathcal{D}$ :

$$
\mathcal{D}=D_{\text {bndry }}^{+}[\mathcal{R}] \cup D_{\text {bndry }}^{-}[\mathcal{R}] .
$$

Suppose that $\mathcal{D}$ is a sub-region on the conformal boundary of the AdS Poincaré chart; then one may define the associated AdS causal wedge

$$
\mathcal{W}=J_{\text {bulk }}^{+}[\mathcal{D}] \cap J_{\text {bulk }}^{-}[\mathcal{D}]
$$

where $J_{\text {bulk }}^{+(-)}$is the future (past) domain of influence on $d+1$-dimensional PAdS - see figure 3. As with $\mathcal{R}$ we say that the causal wedge $\mathcal{W}$ has origin $\bar{x}$ and radius $L$.

The bulk region of any AdS causal wedge is isomorphic to the region of AdS known as the AdS-Rindler wedge [50]. ${ }^{8}$ Thus the bulk of any causal wedge may be covered by the AdS-Rindler coordinate chart which is independent of a particular wedge's radius and

\footnotetext{
${ }^{8}$ The AdS-Rindler wedge is also known as AdS in hyperbolic coordinates and corresponds to the special case of a hyperbolic AdS black hole metric when the geometry is in fact just pure AdS [8].
} 
origin. Of course, the radius and origin of a causal wedge is encoded in the diffeomorphism necessary to transform from Poincaré coordinates to AdS-Rindler coordinates on the wedge. This diffeomorphism induces a distinct conformal transformation on the boundary theory which similarly depends on the radius and origin of the wedge. The details of this bulk diffeomorphism (boundary conformal transformation) is nicely described in [50], and we do not need to repeat them here. For completeness we provide an example of such of a transformation in appendix B. What is relevant for us is the fact that a boundary-to-bulk map for the AdS-Rindler wedge immediately carries over to the more general case of a causal wedge, and so we need only study the former case in detail.

\subsection{The case of the AdS-Rindler wedge}

Without further ado we examine the boundary-to-bulk map for the AdS-Rindler wedge. To keep the notation light we will specialize our presentation to the case of $D=2+1$ bulk dimensions. This also allows a more direct comparison with the recent works [9-11] as well as the classic references $[51,52]$.

In $2+1$ dimensions the AdS-Rindler metric has the line element

$$
d s^{2}=\frac{\ell^{2}}{z^{2}}\left[-\left(1-\frac{z^{2}}{\ell^{2}}\right) d \eta^{2}+\left(1-\frac{z^{2}}{\ell^{2}}\right)^{-1} d z^{2}+d \chi^{2}\right] .
$$

It is useful to introduce a complete set of canonically-normalized bulk Klein-Gordon modes $S_{k}(X)=N_{k} e^{i k \cdot x} V_{k}(z)$ where $k \cdot x=-\omega t+k^{1} \chi$. These modes are normalized such that two modes have Klein-Gordon inner product

$$
4 \pi^{2} \delta\left(\omega_{1}-\omega_{2}\right) \delta\left(k_{1}^{1}-k_{2}^{1}\right)=-\left.i \int_{-\infty}^{\infty} d \chi \int_{0}^{\ell} \frac{d z}{z}\left(1-\frac{z^{2}}{\ell^{2}}\right)^{-1} S_{1}(X) \overleftrightarrow{\partial_{\eta}} S_{2}^{*}(X)\right|_{\eta=\text { const }}
$$

The $z$-dependence is contained in [30]

$$
V_{k}(z)=\left(\frac{z}{\ell}\right)^{\Delta}\left(1-\frac{z^{2}}{\ell^{2}}\right)^{-i \ell \omega / 2}{ }_{2} F_{1}\left[\frac{\Delta-i \ell \omega+i \ell k^{1}}{2}, \frac{\Delta-i \ell \omega-i \ell k^{1}}{2} ; \Delta ; \frac{z^{2}}{\ell^{2}}\right] .
$$

The $V_{k}(z)$ are real, satisfy

$$
V_{\omega, k^{1}}(z)=V_{-\omega, k^{1}}(z)=V_{\omega,-k^{1}}(z)=V_{-\omega,-k^{1}}(z),
$$

and are normalized such that near $z=0$ the modes behave as

$$
V_{k}(z)=\left(\frac{z}{\ell}\right)^{\Delta}\left(1+O\left(z^{2}\right)\right), \quad z \rightarrow 0 .
$$

In order to satisfy (3.13) the normalization constant must be chosen to satisfy ${ }^{9}$

$$
\left|N_{k}\right|^{2}=\frac{1}{2 \ell|\omega|} \frac{\Gamma\left(\frac{\Delta+i \ell\left(\omega+k^{1}\right)}{2}\right) \Gamma\left(\frac{\Delta-i \ell\left(\omega+k^{1}\right)}{2}\right) \Gamma\left(\frac{\Delta+i \ell\left(\omega-k^{1}\right)}{2}\right) \Gamma\left(\frac{\Delta-i \ell\left(\omega-k^{1}\right)}{2}\right)}{\Gamma^{2}(\Delta) \Gamma(i \ell \omega) \Gamma(-i \ell \omega)} .
$$

\footnotetext{
${ }^{9}$ Recall that the Gamma function satisfies $\Gamma(x)^{*}=\Gamma\left(x^{*}\right)$, so it is easy to see that $\left|N_{k}\right|^{2}$ is indeed non-negative.
} 
In this setting there are two natural vacua, each of which is locally analytic on the wedge: the usual global AdS vacuum $\Omega$ and the AdS-Rindler (AdSR) vacuum. The latter is simply the state with zero particles in the particle basis defined by the AdS-Rindler coordinate $\eta$; it has the Wightman 2-pt function

$$
\left\langle\Phi\left(X_{1}\right) \Phi\left(X_{2}\right)\right\rangle_{\mathrm{AdsR}}=\ell \int \frac{d^{2} k}{4 \pi^{2}} \theta(\omega)\left|N_{k}\right|^{2} e^{i k \cdot\left(x_{1}-x_{2}\right)} V_{k}\left(z_{1}\right) V_{k}\left(z_{2}\right) .
$$

This state induces on the boundary the Rindler $(\mathrm{R})$ state whose 2-pt function is easily read off from (3.18):

$$
\left\langle\phi\left(x_{1}\right) \phi\left(x_{2}\right)\right\rangle_{R}=\ell^{2-2 \Delta} \int \frac{d^{2} k}{4 \pi^{2}} \theta(\omega)\left|N_{k}\right|^{2} e^{i k \cdot\left(x_{1}-x_{2}\right)} .
$$

Returning to the bulk, consider now the global AdS vacuum restricted the the AdS-Rindler chart. As is well-known, on this chart the global AdS vacuum satisfies the KMS condition with inverse temperature $\beta=2 \pi \ell$ (in natural units):

$$
\left\langle\Phi\left(\eta_{1}, x_{1}\right) \Phi\left(\eta_{2}, x_{2}\right)\right\rangle_{\Omega}=\left\langle\Phi\left(\eta_{2}, x_{2}\right) \Phi\left(\eta_{1}+i 2 \pi \ell, x_{1}\right)\right\rangle_{\Omega} .
$$

Using standard thermal field theory techniques it is easy to deduce from this expression that the global AdS 2-pt function may be written

$$
\left\langle\Phi\left(X_{1}\right) \Phi\left(X_{2}\right)\right\rangle_{\Omega}=\ell \int \frac{d^{2} k}{4 \pi^{2}} e^{i k \cdot\left(x_{1}-x_{2}\right)} \frac{e^{\pi \ell \omega}}{2|\sinh \pi \ell \omega|}\left|N_{k}\right|^{2} V_{k}\left(z_{1}\right) V_{k}\left(z_{2}\right) .
$$

This state induces the usual Minkowski vacuum state $\omega$ on the boundary: ${ }^{10}$

$$
\left\langle\phi\left(x_{1}\right) \phi\left(x_{2}\right)\right\rangle_{\omega}=\ell^{2-2 \Delta} \int \frac{d^{2} k}{4 \pi^{2}} \frac{e^{\pi \ell \omega}}{2|\sinh \pi \ell \omega|}\left|N_{k}\right|^{2} e^{i k \cdot\left(x_{1}-x_{2}\right)} .
$$

Like $\Omega$ in the bulk $\omega$ is a KMS state with $\beta=2 \pi \ell$. From the expressions above one may easily verify the local analyticity of these states.

We can establish a map $\mathcal{A}_{\text {bndry }}(\mathcal{D}) \mapsto \mathcal{A}_{\text {bulk }}(\mathcal{W})$ just as we did for the Poincaré chart. The mapping kernel $K(X \mid y)$ is easily constructed from the bulk Klein-Gordon modes:

$$
K(X \mid y)=\frac{1}{\sqrt{-\gamma(y)}} \int \frac{d^{2} k}{4 \pi^{2}} e^{i k \cdot(x-y)} V_{k}(z),
$$

where $\sqrt{\gamma(-y)}=\Omega^{2}(y)=e^{2 \chi / \ell}$. As we will see momentarily, the Klein-Gordon modes $V_{k}(z)$ can grow as $|k| \rightarrow \infty$ and so $K(X \mid y)$ must be regarded as a distribution. Formally $K(X \mid y)$ constructs a boundary test function $f_{F}(y)$ for every bulk test function $F(X) \in$ $C_{0}^{\infty}(\mathcal{W})$ as in (3.8). However, recall from the discussion in section 3.1 that boundary test

\footnotetext{
${ }^{10}$ This $\omega$ Wightman function may also be obtained by considering the action of the conformal transformation on the Minkowski vacuum 2-pt function in Cartesian coordinates. Under this conformal transformation the Minkowski 2-pt function becomes

$$
\left\langle\phi\left(x_{1}\right) \phi\left(x_{2}\right)\right\rangle_{\omega}=\frac{1}{2 \pi} \frac{1}{\left(x_{1}-x_{1}\right)^{2 \Delta}} \rightarrow \frac{1}{(2 \pi) 2^{\Delta} \ell^{2 \Delta}}\left(\cosh \left[\frac{\chi_{1}-\chi_{2}}{\ell}\right]-\cosh \left[\frac{\eta_{1}-\eta_{2}}{\ell}\right]\right)^{-\Delta} .
$$

Taking the Fourier transform of this expression yields (3.23).
} 
functions yield well-defined observables only if they are members of the class $\mathscr{T}$. Also recall that $f_{F} \in \mathscr{T}$ only if $f_{F}(y)$ satisfies the wave front set condition (3.4). Thus our task is to determine if the $f_{F}(y)$ constructed using $K(X \mid y)$ have wave front sets which satisfy (3.4).

We can accomplish this by examining the behavior of the Fourier transforms $\hat{f}_{F}(k)$ in AdS-Rindler coordinates. Of course the AdS-Rindler chart is not a locally-flat coordinate chart like the charts involved computing wave front sets, but nevertheless we may infer from the AdS-Rindler Fourier transform sufficient information to determine if $f_{F} \in \mathscr{T}$. To start consider a bulk test function

$$
F(X)=\int \frac{d^{2} k}{4 \pi^{2}} e^{i k \cdot x} \hat{F}_{k}(z), \quad F \in C_{0}^{\infty}(\mathcal{W}) .
$$

Because $F(X)$ is smooth it follows that it's wave front set it empty and we may convolve $F(X)$ with $K(X \mid y)$. The resulting associated boundary test function $f_{F}(y)$ has the AdSRindler Fourier transform

$$
\hat{f}_{F}(k)=\ell^{3} \int_{0}^{\ell} \frac{d z}{z^{3}} \hat{F}_{k}(z) V_{k}(z)
$$

We wish to determine the behavior of $\hat{f}_{F}(k)$ at large $|k|$, and for this we need to know the behavior of $V_{k}(z)$ as $|k| \rightarrow \infty$ in a given direction in momentum space while $\Delta, z$ are held fixed. Examining the equation of motion governing $V_{k}(z)$ one may readily see that this limit corresponds to the limit where the effective mass-squared term becomes large in magnitude. In this regime the asymptotic form of $V_{k}(z)$ may be reliably computed using the WKB approximation. We carry out this analysis in appendix C. For $\omega^{2} \gg\left(k^{1}\right)^{2}, \Delta^{2}$ we obtain the asymptotic form

$$
V_{k}(z) \approx \frac{2^{\Delta-1} \Gamma(\Delta)}{\sqrt{2 \pi}}|\ell \omega|^{1 / 2-\Delta}\left(\frac{z}{\ell}\right)^{1 / 2}\left[\left(\frac{1+z / \ell}{1-z / \ell}\right)^{i \ell \omega / 2}+\left(\frac{1+z / \ell}{1-z / \ell}\right)^{-i \ell \omega / 2}\right]
$$

When $|k| \rightarrow \infty$ along a null direction there is qualitatively similar behavior, i.e. $V_{k}(z)$ is bounded by a power of $|k|$, with oscillatory dependence on $z / \ell$. Finally, for $\left(k^{1}\right)^{2} \gg \omega^{2}, \Delta$ we obtain the asymptotic form

$$
V_{k}(z) \approx C\left|\ell k^{1}\right|^{1-\Delta}\left[\frac{z / \ell}{\ell\left|k^{1}\right|\left(1-(z / \ell)^{2}\right)^{1 / 2}}\right]^{1 / 2}\left(e^{\ell k^{1} \arcsin (z / \ell)}+e^{-\ell k^{1} \arcsin (z / \ell)}\right),
$$

where $C$ is a finite constant independent of $k^{1}$. This expression diverges most strongly as $z \rightarrow \ell$, and from this behavior we bound $V_{k}(z)$ in this regime by

$$
V_{k}(z) \leq C\left|k^{1}\right|^{1 / 2-\Delta} e^{\frac{\pi}{2}\left|k^{1}\right|},
$$

with $C$ another finite constant independent of $k^{1}$. Taken together, these results show that the AdS-Rindler Fourier transform $\hat{f}_{F}(y)$ decays faster than any power as $|k| \rightarrow \infty$ along timelike and null directions (in AdS-Rindler coordinates). This is sufficient to determine that the wave front set of $f_{F}(y)$ satisfies the condition (3.4), and thus that $f_{F} \in \mathscr{T}$.

Of course we do not need fancy wave front set arguments to show that the boundary observables $\phi\left[f_{F}\right]$ have well-defined correlation functions for physically reasonable states. 
One may obtain the same conclusion by direct computation, e.g. by examining the $\phi\left[f_{F}\right]$ correlators of $\omega$ and $R$. Consider the correlator

$$
\left\langle\phi\left(f_{F_{1}}\right) \phi\left(f_{F_{2}}\right)\right\rangle_{R}=\int \frac{d^{2} k}{4 \pi^{2}}\left|N_{k}\right|^{2} \hat{f}_{F_{1}}(-k) \hat{f}_{F_{2}}(k) .
$$

Using the asymptotic formula for the Gamma function (C.14) we readily obtain the limits

$$
\begin{aligned}
& \lim _{\ell\left|k^{1}\right| \rightarrow \infty}\left|N_{k}\right|^{2}=C_{1}\left|\ell k^{1}\right|^{2 \Delta-2} e^{-\pi \ell\left|k^{1}\right|}, \quad \Delta, \omega \text { fixed, } \\
& \lim _{\ell|\omega| \rightarrow \infty}\left|N_{k}\right|^{2}=C_{2}|\ell \omega|^{2 \Delta-2}, \quad \Delta, k^{1} \text { fixed, }
\end{aligned}
$$

with finite constants $C_{1}, C_{2}$. Combining this with the the asymptotic behavior of the Klein-Gordon modes (3.27), (3.28) we see that the Fourier transform of $\left\langle\phi\left(f_{F_{1}}\right) \phi\left(f_{F_{2}}\right)\right\rangle_{R}$ converges absolutely. The story is quite the same for the Minkowski vacuum $\omega$ whose 2-pt function Fourier transform differs only by the addition of a factor $e^{\pi \ell \omega} / \sinh (\pi \ell \omega)$. The story is also the same for states constructed from $\omega$ by acting with $\mathcal{A}_{\text {bndry }}$ as well as states constructed from $R$ by acting with $\mathcal{A}_{\text {bndry }}(\mathcal{D})$.

\section{Discussion}

The goal of this work has been to understand in a precise way how "bulk information" is encoded in the boundary CFT using the simple example of an AdS Klein-Gordon field as a case study. In particular we have been interested in quantifying the information available to a boundary observer with access limited to a sub-region of the boundary. It is useful to describe our results in terms of three processes: reading, writing, and storing information. In section 3 we showed that an observer with access to the observables within domain of dependence $\mathcal{D}$ on the boundary may reconstruct all bulk observables contained within the associated AdS causal wedge anchored to $\mathcal{D}$. We say that this observer may read the information of the bulk algebra of observables $\mathcal{A}_{\text {bulk }}(\mathcal{W})$. Any observer with access to a boundary domain $\mathcal{D}$ whose associated causal wedge contains an observable $A \in \mathcal{A}_{\text {bulk }}$ may read $A$. This set of boundary domains has no mutual overlap. So, while the information of $A$ may be read from several boundary domains, it is inappropriate to say that this information is stored in any of them — see again figure 1.

The ability of a boundary observer to write bulk information is encapsulated in the holographic form of the Reeh-Schlieder theorem:

Let $\mathcal{W}$ be an AdS causal wedge with conformal boundary $\mathcal{D}$. Let $\Psi$ be locally analytic on $\mathcal{W}$ and let $\psi$ be it's boundary value. Then the linear spans of the sets of states

$$
S_{\mathcal{D}}=\left\{A \psi \mid A \in \mathcal{A}_{\text {bndry }}(\mathcal{D})\right\}, \quad S_{\mathcal{W}}=\left\{B \Psi \mid B \in \mathcal{A}_{\text {bulk }}(\mathcal{W})\right\}
$$

satisfy $\operatorname{span}\left(S_{\mathcal{W}}\right) \subset \operatorname{span}\left(S_{\mathcal{D}}\right)$

By now the reader is familiar with the implications of this statement. It we consider the case where $\Psi$ is the AdS vacuum $\Omega$ then the set $S_{\mathcal{D}}$ spans the set of finite-particle states, or more 
generally the set of finite energy-density states which may be constructed from our bulk algebra of observables. If we instead consider the vacuum state of a causal wedge $\mathcal{W}_{2} \supseteq \mathcal{W}$ then set $S_{\mathcal{D}}$ spans the full set of finite-particle states built atop this vacuum. Either way, the point is that the set of observables in $\mathcal{D}$ is powerful enough to construct essentially any state consistent with the bulk semi-classical approximation. In this sense, by careful manipulation of $\mathcal{A}_{\text {bndry }}(\mathcal{D})$ one may write more than one can read. Another interpretation of the bulk RS theorem is that states satisfying reasonable energy conditions are have a high degree of entanglement. In holography we may exploit this entanglement to control the bulk system using only a sub-region of the boundary. We remind the reader that we are considering here just the effects of acting with normalizable excitations at the boundary.

At first glance our formulation of the Reeh-Schlieder theorem appears somewhat weaker than that of Minkowski QFT found in textbooks [2, 47]. In textbook accounts the RS theorem states the the set of states generated from the vacuum by a sub-set of the observable algebra is dense on the Hilbert space. Our theorem is phrased in terms of linear spans of sets of states generated by a $*$-algebra. The added power of the textbook theorems comes from additional technical assumptions about the Hilbert space which follow from, e.g., adopting the Wightman axioms as in the original work [1] or some suitable generalization for curved spacetimes [48]. We feel that our phrasing gets to the point of the RS theorem without boring the reader with too many technical details. That said, we are happy to point out a few technical assumptions of our analysis which could be violated so as to nullify our conclusions:

1. We consider $*$-algebras of observables which have no norm. Using these algebras we cannot generate an infinite particle state from a state with finitely many particles, nor can we construct compactly-supported unitary operators. The latter are especially useful for avoiding the conclusions of the RS theorem. In order to describe these objects consistently one has do to more work and specify a norm for the algebras (equivalently, a norm for the Hilbert space).

2. We restrict attention to states which may be generated from locally analytic states. We are motivated to do so because we know that these states have well-behaved average stress-energy fluctuations. Of course one could consider other, more singular states.

It is natural to ask how our analysis might be extended to more interesting bulk geometries. To make this discussion as interesting as possible let us consider asymptotically-AdS wormhole geometries [53-57], an example of which is depicted in figure 4. These geometries have regions of the bulk with no causal contact with the boundaries. We continue to operate within the semi-classical approximation where we have a known, fixed bulk geometry and would like to recover the bulk observables of a bulk scalar field given only it's boundary correlation functions. First consider recovery for bulk observables in the regions exterior to the wormhole (the white regions in figure 4). Given our analysis of bulk-to-boundary maps in exact AdS, along with the many excellent previous works on this subject [28-36], we see no credible obstacle to constructing such a map between a suitable algebra of boundary 


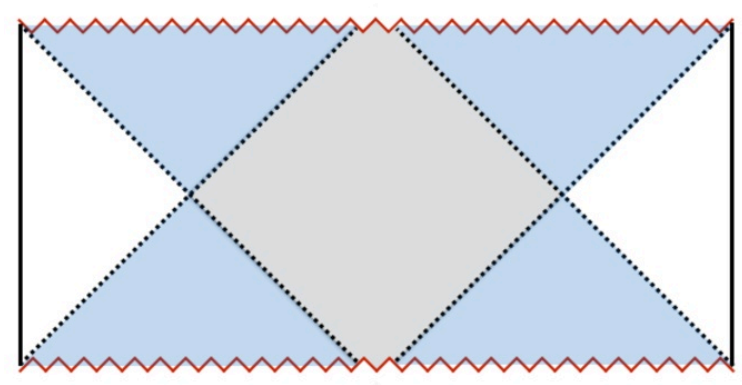

Figure 4. Conformal diagram of a simple AdS wormhole geometry. There are two disconnected conformal boundaries. Each blue shaded region has causal contact with the boundary only through one of it's past or future domains of influence. The gray shaded region has no causal contact with the boundaries.

observables and the algebra of bulk observables restricted to these exterior regions. But such a map does not tell us about the quantum state beyond the horizon; for this we need additional ingredients.

Recently some works $[58,59]$ which consider asymptotically-AdS black holes have advocated analytic continuation as a possible tool for extending a state defined in the exterior region to the region past the horizon. Our concern with this approach is that such an analytic continuation does not necessarily provide the unique extension of a state. As we mentioned when discussing locally analytic states in AdS, it is very rare to have a unique notion of analytic continuation for quantum states. Even in AdS very few states could be called analytic from any reasonable perspective. Leaving aside their distributional aspects, the correlation functions of typical well-behaved quantum states are at best smooth, and there exist many ways to extend a smooth solution to a local equation of motion from a given domain into it's causal complement. In this respect we sympathize with [60]: proving the existence of a smooth extension of correlation functions past the horizon is not the same thing a showing that the physical state in question corresponds to this extension.

Perhaps a more promising approach is to take advantage of the additional information available to us if we have access to a complete Cauchy surface of the boundary theory. In this case we have access to any global charge of the bulk theory which satisfies a Gauss' Law type conservation law. As has been emphasized by many before [61-65], theories with dynamical gravity and stringy excitations necessarily have such charges, including the bulk gravitational Hamiltonian. Within the semi-classical setting one can imagine toy quantum field theories on AdS wormhole geometries for which there exist enough Gauss' law-type charges to completely determine the bulk quantum state. It would be very interesting to study such models in detail.

\section{Acknowledgments}

We thank Madeline Anthonisen, Robert Brandenberger, Daniel Kabat, Alex Maloney, Guy Moore, Matthew Roberts, Vladimir Rosenhaus, and Aron Wall for useful conversations. 


\section{A Wave front sets}

In this appendix we provide a brief introduction to the wave front set of a distribution, following closely the standard reference [66] as well as the introduction contained in [45]. We denote the set of smooth functions on a manifold $M$ by $\mathcal{E}(M)$ and the corresponding dual space of distributions $\mathcal{E}^{\prime}(M)$. We are interested in characterizing the singularity structure of a distribution $u \in \mathcal{E}^{\prime}(M)$. For the moment we consider $M=\mathbb{R}^{n}$. We denote the Fourier transform of $u$ by $\hat{u}$, and we refer to the dual variables of the Fourier transform as position $x$ and momentum $k$ respectively.

The most basic measure of singularity in position space is the singular support of $u$, denoted $\operatorname{singsupp}(u)$, which is the set of points in $\mathbb{R}^{n}$ having no open neighborhood to which the restriction of $u$ is a smooth function. The singular support describes where in position space a distribution is singular, but in no way describes "how" it is singular.

We may also describe the singular nature of a distribution in momentum space. Recall that the Fourier transform of a smooth function $f \in \mathcal{E}\left(\mathbb{R}^{n}\right)$ decays faster than any power law at large momenta:

$$
|\hat{f}(k)| \leq \frac{C_{N}}{(1+|k|)^{N}}, \quad N \in \mathbb{N}_{0},
$$

for some finite constants $C_{N}$. Unless $u$ is in fact induced by a smooth function $\hat{u}$ will fail the bound (A.1) in some directions of momentum space. We may thus define the singular cone of $u$, denoted $\Sigma(u)$, as the conic set ${ }^{11}$ of all $k \in \mathbb{R}^{n} \backslash\{0\}$ having no conic neighborhood for which (A.1) is valid. The singular cone describes the "singular directions" in momentum space for which $\hat{u}$ fails to behave like the Fourier transform of a smooth function.

$\mathrm{N}$ every direction $k \in \Sigma(u)$ is effectively problematic at a given point $x \in \operatorname{singsupp}(u)$. In order to determine which singular directions are the culprits of a given singularity we need a more refined, local notion of the singular directions at a given point. A basic property of the singular cone is that

$$
\Sigma(f u) \subseteq \Sigma(u), \quad f \in \mathcal{E}\left(\mathbb{R}^{n}\right) .
$$

This gives us a natural way to define the cone of singular directions at a point $x$ :

$$
\Sigma_{x}(u):=\cap_{f} \Sigma(f u), \quad f \in \mathcal{E}\left(\mathbb{R}^{n}\right), \quad f(x) \neq 0 .
$$

We call the pair $\left(x_{0}, k_{0}\right) \in \mathbb{R}^{n} \times\left(\mathbb{R}^{n} \backslash\{0\}\right)$ a singular directed point of $u$ if $x_{0} \in \operatorname{singsupp}(u)$ and $k_{0} \in \Sigma_{x_{0}}(u)$. The set of singular directed points of $u$ is it's wave front set:

Definition A.1 The wave front set of a distribution $u \in \mathcal{E}^{\prime}\left(\mathbb{R}^{n}\right)$ is

$$
\mathrm{WF}(u):=\left\{(x, k) \in \mathbb{R}^{n} \times\left(\mathbb{R}^{n} \backslash\{0\}\right) \mid k \in \Sigma_{x}(u)\right\} .
$$

The wave front set is a conic set with respect to the momentum variable. The projection of $\mathrm{WF}(u)$ in position space is $\operatorname{singsupp}(u)$; the projection of $\mathrm{WF}(u)$ in momentum space is $\Sigma(u)$. The wave front set cannot be enlarged by convolution with a smooth function,

$$
\mathrm{WF}(f u) \subseteq \mathrm{WF}(u), \quad f \in \mathcal{E}\left(\mathbb{R}^{n}\right),
$$

\footnotetext{
${ }^{11}$ A conic set $V \subset \mathbb{R}^{n} \backslash\{0\}$ is a set of points containing $k$ as well as all points $c k$ for all constants $c>0$.
} 
or by a derivative operator $\partial$ :

$$
\mathrm{WF}(\partial u) \subset \mathrm{WF}(u) .
$$

Example A.2 The Dirac delta function $\delta(x)$ on $\mathbb{R}^{n}$ has the wave front set

$$
\mathrm{WF}(\delta)=\left\{(x, k) \in \mathbb{R}^{n} \times \mathbb{R}^{n} \backslash\{0\} \mid x=0\right\} .
$$

The wave front set provides a precise criteria for when the point-wise product of a pair of distributions is well-defined (cf. Theorem 8.2.10 of [66]). The point-wise product uv of two distributions $u, v$ is uniquely defined unless there exists a singular directed point $(x, k)$ such that $(x, k) \in \mathrm{WF}(u)$ and $(x,-k) \in \mathrm{WF}(v)$. Roughly speaking, this criteria states that the convolution of distributions unambiguously defines a new distribution so long as singular directed points of do not overlap in convolution.

We have been discussing the $C^{\infty}$ wave front set which describes the failure of a distribution to be smooth. One can similarly define an analytic wave front set $\left(\mathrm{WF}_{A}\right)$ which describes the failure of a distribution to be analytic. For details see [66]. Obviously, $\mathrm{WF}(u) \subset \mathrm{WF}_{\mathrm{A}}(u)$.

The wave front set generalizes quite easily to distributions on manifolds. To see this note that both the singular support and the singular cone at $x$ are locally defined, and thus the wave front set is a local concept. It can be shown that the wave front set transforms covariantly under diffeomorphisms. Taken together these properties indicate that the wave front set is properly thought of as a conic subset of the cotangent bundle, i.e. WF $\subset$ $T^{*} M \backslash\{0\}$. The properties of the wave front set described above for above hold as well for $u \in \mathcal{E}^{\prime}(M), f \in \mathcal{E}(M)$, where $M$ is an arbitrary manifold. Further details of wave front sets for distributions on manifolds may be found in, e.g., [45, 67, 68].

Example A.3 Consider a CFT on $\mathbb{R}^{d, 1}$. The 2-pt function of a scalar CFT operator of weight $\Delta$ with respect to the CFT vacuum is given by

$$
W\left(x_{1}, x_{2}\right) \propto\left(x_{1}-x_{2}\right)^{-2 \Delta},
$$

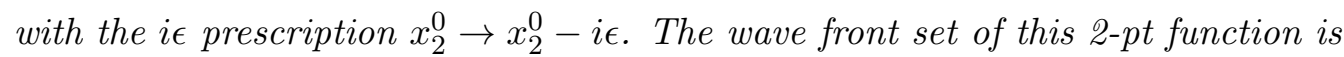

$$
\mathrm{WF}(W)=\left\{\left(x_{1}, k_{1} ; x_{2},-k_{1}\right) \in\left(T^{*}\left(\mathbb{R}^{d, 1}\right)\right)^{2} \backslash\{0\} \mid\left(x_{1}-x_{2}\right)^{2}=0, k_{1} \in V^{+}\right\} .
$$

The closed forward lightcone $V^{+}$is defined below equation (2.9).

\section{B An example transformation to an AdS causal wedge}

In this appendix we give an example of the bulk diffeomorphism (boundary conformal transformation) which establishes an AdS-Rindler chart on an AdS causal wedge. Although the procedure is the same in all bulk dimensions $D=d+1 \geq 3$ the notation is least cumbersome if we restrict to $D=2+1$ bulk dimensions. 
Recall that $\mathrm{AdS}_{3}$ may be defined as the single-sheet hyperbaloid of radius $\ell$ in a $\mathbb{R}^{2,2}$ embedding space

$$
\mathrm{AdS}_{3}:=\left\{\mathcal{X} \in \mathbb{R}^{2,2} \mid-\left(\mathcal{X}^{-1}\right)^{2}-\left(\mathcal{X}^{0}\right)^{2}+\left(\mathcal{X}^{1}\right)^{2}+\left(\mathcal{X}^{2}\right)^{2}=-\ell^{2}\right\} .
$$

The embedding coordinates $\mathcal{X}$ provide a very useful way of relating AdS coordinate charts. The Poincaré chart (2.1) may be related to the embedding coordinates via

$$
\begin{aligned}
\mathcal{X}^{-1} & =\ell \frac{T}{Z}, \\
\mathcal{X}^{0} & =\frac{1}{2 Z}\left(-T^{2}+Y^{2}+Z^{2}+\ell^{2}\right), \\
\mathcal{X}^{1} & =\frac{1}{2 Z}\left(T^{2}-Y^{2}-Z^{2}+\ell^{2}\right), \\
\mathcal{X}^{2} & =\ell \frac{Y}{Z}
\end{aligned}
$$

and the AdS-Rindler chart (3.12) is related to the embedding coordinates via

$$
\begin{aligned}
\mathcal{X}^{-1} & =\frac{\ell^{2}}{z}\left(1-\frac{z^{2}}{\ell^{2}}\right)^{1 / 2} \sinh \frac{\eta}{\ell} \\
\mathcal{X}^{0} & =\frac{\ell^{2}}{z} \cosh \frac{\chi}{\ell} \\
\mathcal{X}^{1} & =-\frac{\ell^{2}}{z} \sinh \frac{\chi}{\ell}, \\
\mathcal{X}^{2} & =\frac{\ell^{2}}{z}\left(1-\frac{z^{2}}{\ell^{2}}\right)^{1 / 2} \cosh \frac{\eta}{\ell} .
\end{aligned}
$$

In the embedding space description the AdS isometries are the subset of isometries of $\mathbb{R}^{2,2}$ which preserve the hyperbaloid, i.e. they are the boosts and rotations of $\mathbb{R}^{2,2}$. Consider a boost in the $\mathcal{X}^{0}-\mathcal{X}^{2}$ plane. Although this is an AdS isometry it does not preserve the AdSRindler chart (B.3). Thus, for each boost parameter $\beta$ we may define boosted embedding coordinates $\mathcal{X}(\beta)$ and then a new AdS-Rindler chart defined as in (B.3) but with $\mathcal{X}$ on the left-hand side replaced by $\mathcal{X}(\beta)$. Relating this new AdS-Rindler chart to the original embedding coordinates $\mathcal{X}$ we obtain

$$
\begin{aligned}
\mathcal{X}^{-1} & =\frac{\ell^{2}}{z}\left(1-\frac{z^{2}}{\ell^{2}}\right)^{1 / 2} \sinh \frac{\eta}{\ell}, \\
\mathcal{X}^{0} & =\frac{\ell^{2}}{z}\left[\cosh \beta \cosh \frac{\chi}{\ell}+\left(1-\frac{z^{2}}{\ell^{2}}\right)^{1 / 2} \sinh \beta \cosh \frac{\eta}{\ell}\right], \\
\mathcal{X}^{1} & =-\frac{\ell^{2}}{z} \sinh \frac{\chi}{\ell}, \\
\mathcal{X}^{2} & =\frac{\ell^{2}}{z}\left[\sinh \beta \cosh \frac{\chi}{\ell}+\left(1-\frac{z^{2}}{\ell^{2}}\right)^{1 / 2} \cosh \beta \cosh \frac{\eta}{\ell}\right] .
\end{aligned}
$$

One may now use (B.2) to relate the coordinates defined on the right-hand side of (B.4) to the original Poincaré chart. The resulting expressions for $(T, Y, Z)$ in terms of $(\eta, \chi, z)$ are 
complicated; what is important is to note that near the boundary $z \rightarrow 0$ these relations are

$$
\begin{aligned}
T & =\frac{\ell \sinh \frac{\eta}{\ell}}{\cosh \beta \cosh \frac{\chi}{\ell}+\sinh \beta \cosh \frac{\eta}{\ell}-\sinh \frac{\chi}{\ell}}+O\left(z^{2}\right), \\
Y & =\frac{\ell\left(\cosh \beta \cosh \frac{\eta}{\ell}+\sinh \beta \cosh \frac{\chi}{\ell}\right)}{\cosh \beta \cosh \frac{\chi}{\ell}+\sinh \beta \cosh \frac{\eta}{\ell}-\sinh \frac{\chi}{\ell}}+O\left(z^{2}\right) .
\end{aligned}
$$

Thus, after converting bulk coordinates from Poincaré to the boosted AdS-Rindler system the boundary metric takes the form

$$
d s_{\text {bndry }}^{2}=\Omega^{2}(x)\left(-d \eta^{2}+d \chi^{2}\right)
$$

where the conformal factor is

$$
\Omega(x)=\frac{1}{\cosh \beta \cosh \frac{\chi}{\ell}+\sinh \beta \cosh \frac{\eta}{\ell}-\sinh \frac{\chi}{\ell}} .
$$

This new boundary chart covers a domain of dependence $\mathcal{D}$ with origin $\bar{x}=(0, \ell \operatorname{coth} \beta)$ and radius $L=\ell \operatorname{csch} \beta$. In the bulk the horizon at $z=\ell$ corresponds precisely to the edge of the associated spherical causal wedge. In a similar fashion one may use a second boost in the embedding space to shift the location of the origin of the causal wedge.

\section{WKB approximations of AdS-Rindler Klein-Gordon modes}

Here we derive the WKB approximations for the AdS-Rindler Klein-Gordon mode functions quoted in section 3.4. In AdS-Rindler coordinates (3.12) the scalar d'Alembertian is

$$
\square \Phi(X)=\frac{z^{2}}{\ell^{2}}\left[-\left(1-\frac{z^{2}}{\ell^{2}}\right)^{-1} \partial_{\eta}^{2}+\partial_{\chi}^{2}+\left(1-\frac{z^{2}}{\ell^{2}}\right) \partial_{z}^{2}-\left(1-\frac{z^{2}}{\ell^{2}}\right) \frac{1}{z} \partial_{z}\right] \Phi(X) .
$$

Using this and the form of the Klein-Gordon modes $e^{i k \cdot x} V_{k}(z)$ we obtain the equation satisfied by the radial function $V_{k}(z)$. To keep the notation manageable we adopt the dimensionless variables $\hat{z}=z / \ell, \hat{k}^{1}=\ell k^{1}$, and $\hat{\omega}=\ell \omega$. Then the equation for $V_{k}(z)$ may be written

$$
\left(1-\hat{z}^{2}\right) V_{k}^{\prime \prime}(\hat{z})-\frac{1+\hat{z}^{2}}{\hat{z}} V_{k}^{\prime}(\hat{z})-\mathcal{M}^{2}(\hat{z}) V_{k}(\hat{z})=0,
$$

where we have defined the effective mass-squared

$$
\mathcal{M}^{2}(\hat{z})=\frac{\Delta(\Delta-2)}{\hat{z}^{2}}+\left(\hat{k}^{1}\right)^{2}-\frac{\hat{\omega}^{2}}{1-\hat{z}^{2}} .
$$

We are interested in three regimes:

- case i): the large frequency regime

$$
\hat{\omega}^{2} \gg\left(1-\hat{z}^{2}\right)\left[\frac{\Delta(\Delta-2)}{\hat{z}^{2}}+\left(\hat{k}^{1}\right)^{2}\right],
$$


- case ii): the large spatial momentum regime

$$
\left(\hat{k}^{1}\right)^{2} \gg \frac{\hat{\omega}^{2}}{1-\hat{z}^{2}}-\frac{\Delta(\Delta-2)}{\hat{z}^{2}},
$$

and

- case iii): large null momentum, e.g., for $\hat{k}^{ \pm}=\hat{\omega} \pm \hat{k}^{1}$

$$
\frac{\left(\hat{k}^{+}\right)^{2}}{4} \gg-\frac{\left(\hat{k}^{-}\right)^{2}}{4}+\frac{\left(1-\hat{z}^{2}\right)}{\hat{z}^{2}}\left[\frac{\Delta(\Delta-2)}{\hat{z}^{2}}+O\left(\hat{k}^{ \pm}\right)\right] .
$$

For each of these regimes the effective mass-squared $\left|\mathcal{M}^{2}(\hat{z})\right| \gg 1$, so we may use a WKB analysis to obtain an approximate solution. We anticipate a solution of the form

$$
V(\hat{z})=K e^{A(\hat{z})},
$$

where $A(\hat{z})=A_{0}(\hat{z})+A_{1}(\hat{z})+\ldots$ contains successive terms suppressed by a large factor $\sim \mathcal{M}(\hat{z})$. Under this assumption we may insert (C.7) into (C.2) and solve order-by-order for the $A_{i}(\hat{z})$. At lowest order only the term $\left(A_{0}^{\prime}(\hat{z})\right)^{2}$ contributes and we obtain

$$
A_{0}^{\prime}(\hat{z})= \pm \frac{\mathcal{M}(\hat{z})}{\left(1-\hat{z}^{2}\right)^{1 / 2}}
$$

where $\mathcal{M}(\hat{z})$ may be real or imaginary depending on the regime of interest. At the next order we determine that

$$
A_{1}(\hat{z})=\frac{1}{2} \ln \left[ \pm \frac{\hat{z}}{\left(1-\hat{z}^{2}\right)^{1 / 2} \mathcal{M}(\hat{z})}\right]
$$

To proceed further we specialize for each case:

Case i): Here the frequency term dominates the effective mass-squared, making the mass imaginary:

$$
\mathcal{M}(\hat{z}) \approx \pm i \frac{\omega}{\sqrt{1-\hat{z}^{2}}}
$$

Inserting this into the results above, and integrating to obtain $A_{0}(\hat{z})$, we have

$$
A_{0}(\hat{z})= \pm i \frac{\hat{\omega}}{2} \ln \left[\frac{1+\hat{z}}{1-\hat{z}}\right], \quad A_{1}(\hat{z})=\frac{1}{2} \ln \left[ \pm i \frac{\hat{z}}{\hat{\omega}}\right]
$$

Thus our approximate solution in this regime is

$$
V_{k}(\hat{z}) \approx\left(\frac{\hat{z}}{|\hat{\omega}|}\right)^{1 / 2}\left[K_{k}\left(\frac{1+\hat{z}}{1-\hat{z}}\right)^{i \hat{\omega} / 2}+K_{k}^{*}\left(\frac{1+\hat{z}}{1-\hat{z}}\right)^{-i \hat{\omega} / 2}\right] .
$$

We have combined solutions so that $V_{k}(\hat{z}) \in \mathbb{R}$.

The accuracy of our WKB approximation is governed by the ratio $\left|A_{1}(\hat{z}) / A_{0}(\hat{z})\right|$ (as well as similar expressions at higher orders). From the results above we see that this ratio is suppressed by a large factor $|\ln \hat{\omega} / \hat{\omega}|$ as expected, and thus the WKB approximation is 
valid as $\hat{\omega}^{2} \rightarrow \infty$ for fixed $\Delta, \hat{k}^{1}$, and $\hat{z}$. However, $\left|A_{1}(\hat{z}) / A_{0}(\hat{z})\right|$ is also $\hat{z}$-dependent. For parametrically small $\hat{z} \sim \hat{\omega}^{-1}$ this ratio becomes of order unity and the WKB approximation breaks down. On the bright side, for $\hat{z}$ above this threshold the WKB approximation is valid and even becomes exact as $\hat{z} \rightarrow 1\left(\left|A_{1}(\hat{z}) / A_{0}(\hat{z})\right| \rightarrow 0\right.$ as $\left.\hat{z} \rightarrow 1\right)$. Therefore the approximate solution (C.12) can be matched to the exact solution in this limit, and in this way we can determine the coefficient $K_{k}$. For $\hat{z}=1-\epsilon$ with $\epsilon \ll 1$ the exact result is

$$
V_{k}(\hat{z}=1-\epsilon)=\left[\frac{\Gamma(\Delta) \Gamma(i \hat{\omega})}{\Gamma\left(\frac{\Delta+i \hat{\omega}+i \hat{k}^{1}}{2}\right) \Gamma\left(\frac{\Delta+i \hat{\omega}-i \hat{k}^{1}}{2}\right)} \epsilon^{-i \hat{\omega} / 2}+(\hat{\omega} \rightarrow-\hat{\omega})\right](1+O(\epsilon)) \cdot(
$$

Using this expression and the asymptotic form of the Gamma function

$$
\lim _{|y| \rightarrow \infty} \Gamma(x+i y)=(2 \pi)^{1 / 2} e^{-(\pi / 2)|y|}|y|^{x-1 / 2}\left(1+O\left(y^{-1}\right)\right),
$$

we determine that the coefficient $K_{k}$ is

$$
K_{k}=K_{k}^{*}=\frac{2^{\Delta-1} \Gamma(\Delta)}{\sqrt{2 \pi}}|\hat{\omega}|^{1-\Delta},
$$

as quoted in the text.

Note that one could also determine the $\hat{\omega}$ dependence of the coefficient by matching the WKB approximation to the exact solution in the region $\hat{z} \sim \hat{\omega}^{-1}$. The lack of precision in this matching is reflected by the fact that we can only determine the constant up to an $O(1)$ coefficient independent of $\hat{\omega}$. Through this approach one obtains $K_{k}=C|\hat{\omega}|^{1-\Delta}$, which is of course consistent with the (more accurate) result found above.

Case ii): Now we specialize to case the large momentum regime for which

$$
\mathcal{M}(\hat{z}) \approx \pm \hat{k}^{1}
$$

Then

$$
A_{0}(\hat{z})= \pm \hat{k}^{1} \arcsin \hat{z}, \quad A_{1}(\hat{z})=\frac{1}{2} \ln \left[ \pm \frac{\hat{z}}{\left(1-\hat{z}^{2}\right)^{1 / 2} \hat{k}^{1}}\right],
$$

and our approximate solution becomes

$$
V_{k}(\hat{z}) \approx K_{k}\left[\frac{\hat{z}}{\left|\hat{k}^{1}\right|\left(1-\hat{z}^{2}\right)^{1 / 2}}\right]^{1 / 2}\left(e^{\hat{k}^{1} \arcsin \hat{z}}+e^{-\hat{k}^{1} \arcsin \hat{z}}\right) .
$$

The ratio $\left|A_{1}(\hat{z}) / A_{0}(\hat{z})\right|$ that controls the accuracy of this approximation is suppressed by a factor of $\left|\ln \hat{k}^{1} / \hat{k}^{1}\right|$ and so the approximation is good for fixed $\Delta, \hat{z}$. The $\hat{z}$-dependence of the ratio causes it to become order unity when $\hat{z} \sim\left(\hat{k}^{1}\right)^{-1}$. We may therefore match (C.18) to the exact $V_{k}(z)$ in this region and determine that

$$
K_{k}=C\left|\hat{k}^{1}\right|^{1-\Delta}
$$


for constant $C$ independent of $\hat{\omega}$ and $\hat{k}^{1}$. We note that the WKB approximation formally breaks down when $1-\hat{z} \sim \exp \left[-c\left|\hat{k}^{1}\right|\right]$ as the ratio $\left|A_{1}(\hat{z}) / A_{0}(\hat{z})\right|$ once again becomes order unity, but despite this as far as the dependence on $\hat{k}^{1}$ is concerned there is excellent agreement between (C.18) and (C.13).

Case iii): Finally we describe when $|k| \rightarrow \infty$ is taken along a null direction. This case is qualitatively similar to the large frequency regime (i). Consider when $\left|\hat{k}^{+}\right| \gg\left|\hat{k}^{-}\right|, \Delta, \hat{z}$; then the effective mass is

$$
\mathcal{M}(\hat{z}) \approx \pm \frac{i}{2} \frac{\hat{z} \hat{k}^{+}}{\sqrt{1-\hat{z}^{2}}}
$$

and so

$$
A_{0}(\hat{z})= \pm i \frac{\hat{k}^{+}}{4} \ln \left(1-\hat{z}^{2}\right), \quad A_{1}(\hat{z})=-\frac{1}{2} \ln \left[\mp i \frac{\hat{k}^{+}}{2}\right]
$$

and our WKB approximation is

$$
V(\hat{z}) \approx\left|\hat{k}^{+}\right|^{-1 / 2}\left[K_{k}\left(1-\hat{z}^{2}\right)^{+i k^{+} / 4}+K_{k}^{*}\left(1-\hat{z}^{2}\right)^{-i k^{+} / 4}\right] .
$$

The ratio $\left|A_{1}(\hat{z}) / A_{0}(\hat{z})\right| \rightarrow 0$ as $\hat{z} \rightarrow 1$ indicating exact agreement in this limit, so we match (C.22) with the exact expression (C.13) to determine the coefficient

$$
K_{k}=\frac{2^{\Delta / 2} \Gamma(\Delta)}{\Gamma\left(\frac{\Delta+i \hat{k}^{-}}{2}\right)}\left|\hat{k}^{+}\right|^{(1-\Delta) / 2} .
$$

Open Access. This article is distributed under the terms of the Creative Commons Attribution License (CC-BY 4.0), which permits any use, distribution and reproduction in any medium, provided the original author(s) and source are credited.

\section{References}

[1] S. Schlieder, Some Remarks about the Localization of States in a Quantum Field Theory, Comm. Math. Phys. 1 (1965) 265.

[2] R. Haag, Local quantum physics: fields, particles, algebras. Texts and monographs in physics, Springer-Verlag, Germany (1992).

[3] O. Aharony, S.S. Gubser, J.M. Maldacena, H. Ooguri and Y. Oz, Large-N field theories, string theory and gravity, Phys. Rept. 323 (2000) 183 [hep-th/9905111] [INSPIRE].

[4] E. D'Hoker and D.Z. Freedman, Supersymmetric gauge theories and the AdS /CFT correspondence, hep-th/0201253 [INSPIRE].

[5] G.T. Horowitz and J. Polchinski, Gauge/gravity duality, gr-qc/0602037 [INSPIRE].

[6] B. Czech, J.L. Karczmarek, F. Nogueira and M. Van Raamsdonk, The Gravity Dual of a Density Matrix, Class. Quant. Grav. 29 (2012) 155009 [arXiv:1204.1330] [InSPIRE].

[7] R. Bousso, S. Leichenauer and V. Rosenhaus, Light-sheets and AdS/CFT, Phys. Rev. D 86 (2012) 046009 [arXiv:1203.6619] [INSPIRE].

[8] R. Emparan, AdS/CFT duals of topological black holes and the entropy of zero energy states, JHEP 06 (1999) 036 [hep-th/9906040] [INSPIRE]. 
[9] B. Czech, J.L. Karczmarek, F. Nogueira and M. Van Raamsdonk, Rindler Quantum Gravity, Class. Quant. Grav. 29 (2012) 235025 [arXiv:1206.1323] [INSPIRE].

[10] M. Parikh and P. Samantray, Rindler-AdS/CFT, arXiv:1211.7370 [INSPIRE].

[11] R. Bousso, B. Freivogel, S. Leichenauer, V. Rosenhaus and C. Zukowski, Null Geodesics, Local CFT Operators and AdS/CFT for Subregions, Phys. Rev. D 88 (2013) 064057 [arXiv: 1209.4641] [INSPIRE].

[12] S. Ryu and T. Takayanagi, Holographic derivation of entanglement entropy from AdS/CFT, Phys. Rev. Lett. 96 (2006) 181602 [hep-th/0603001] [INSPIRE].

[13] V.E. Hubeny, M. Rangamani and T. Takayanagi, A Covariant holographic entanglement entropy proposal, JHEP 07 (2007) 062 [arXiv:0705.0016] [INSPIRE].

[14] A. Lewkowycz and J. Maldacena, Generalized gravitational entropy, JHEP 08 (2013) 090 [arXiv: 1304.4926] [INSPIRE].

[15] T. Faulkner, A. Lewkowycz and J. Maldacena, Quantum corrections to holographic entanglement entropy, JHEP 11 (2013) 074 [arXiv: 1307.2892] [INSPIRE].

[16] V.E. Hubeny and M. Rangamani, Causal Holographic Information, JHEP 06 (2012) 114 [arXiv: 1204.1698] [INSPIRE].

[17] H. Casini and M. Huerta, Remarks on the entanglement entropy for disconnected regions, JHEP 03 (2009) 048 [arXiv: 0812.1773] [INSPIRE].

[18] P. Hayden, M. Headrick and A. Maloney, Holographic Mutual Information is Monogamous, Phys. Rev. D 87 (2013) 046003 [arXiv:1107.2940] [INSPIRE].

[19] I.A. Morrison and M.M. Roberts, Mutual information between thermo-field doubles and disconnected holographic boundaries, arXiv:1211.2887 [INSPIRE].

[20] A.C. Wall, Maximin Surfaces and the Strong Subadditivity of the Covariant Holographic Entanglement Entropy, arXiv:1211.3494 [INSPIRE].

[21] T. Hartman and J. Maldacena, Time Evolution of Entanglement Entropy from Black Hole Interiors, JHEP 05 (2013) 014 [arXiv: 1303.1080] [INSPIRE].

[22] D.D. Blanco, H. Casini, L.-Y. Hung and R.C. Myers, Relative Entropy and Holography, JHEP 08 (2013) 060 [arXiv: 1305.3182] [INSPIRE].

[23] W.R. Kelly and A.C. Wall, Coarse-grained entropy and causal holographic information in AdS/CFT, JHEP 03 (2014) 118 [arXiv:1309.3610] [INSPIRE].

[24] P. Kraus, H. Ooguri and S. Shenker, Inside the horizon with AdS/CFT, Phys. Rev. D 67 (2003) 124022 [hep-th/0212277] [INSPIRE].

[25] L. Fidkowski, V. Hubeny, M. Kleban and S. Shenker, The Black hole singularity in AdS/CFT, JHEP 02 (2004) 014 [hep-th/0306170] [INSPIRE].

[26] V.E. Hubeny, H. Liu and M. Rangamani, Bulk-cone singularities $\mathcal{E} 3$ signatures of horizon formation in AdS/CFT, JHEP 01 (2007) 009 [hep-th/0610041] [INSPIRE].

[27] V.E. Hubeny, Extremal surfaces as bulk probes in AdS/CFT, JHEP 07 (2012) 093 [arXiv: 1203.1044] [INSPIRE].

[28] I. Bena, On the construction of local fields in the bulk of $A d S_{5}$ and other spaces, Phys. Rev. D 62 (2000) 066007 [hep-th/9905186] [INSPIRE]. 
[29] A. Hamilton, D.N. Kabat, G. Lifschytz and D.A. Lowe, Local bulk operators in AdS/CFT: A Boundary view of horizons and locality, Phys. Rev. D 73 (2006) 086003 [hep-th/0506118] [INSPIRE].

[30] A. Hamilton, D.N. Kabat, G. Lifschytz and D.A. Lowe, Holographic representation of local bulk operators, Phys. Rev. D 74 (2006) 066009 [hep-th/0606141] [InSPIRE].

[31] A. Hamilton, D.N. Kabat, G. Lifschytz and D.A. Lowe, Local bulk operators in AdS/CFT: A Holographic description of the black hole interior, Phys. Rev. D 75 (2007) 106001 [Erratum ibid. D 75 (2007) 129902] [hep-th/0612053] [INSPIRE].

[32] D.A. Lowe and S. Roy, Holographic description of asymptotically $A d S_{2}$ collapse geometries, Phys. Rev. D 78 (2008) 124017 [arXiv:0810.1750] [INSPIRE].

[33] D. Kabat, G. Lifschytz and D.A. Lowe, Constructing local bulk observables in interacting AdS/CFT, Phys. Rev. D 83 (2011) 106009 [arXiv:1102.2910] [INSPIRE].

[34] I. Heemskerk, Construction of Bulk Fields with Gauge Redundancy, JHEP 09 (2012) 106 [arXiv: 1201.3666] [INSPIRE].

[35] D. Kabat, G. Lifschytz, S. Roy and D. Sarkar, Holographic representation of bulk fields with spin in AdS/CFT, Phys. Rev. D 86 (2012) 026004 [arXiv:1204.0126] [InSPIRE].

[36] D. Kabat and G. Lifschytz, CFT representation of interacting bulk gauge fields in AdS, Phys. Rev. D 87 (2013) 086004 [arXiv:1212.3788] [INSPIRE].

[37] S. Leichenauer and V. Rosenhaus, AdS black holes, the bulk-boundary dictionary and smearing functions, Phys. Rev. D 88 (2013) 026003 [arXiv: 1304.6821] [INSPIRE].

[38] P. Breitenlohner and D.Z. Freedman, Positive Energy in anti-de Sitter Backgrounds and Gauged Extended Supergravity, Phys. Lett. B 115 (1982) 197 [INSPIRE].

[39] P. Breitenlohner and D.Z. Freedman, Stability in Gauged Extended Supergravity, Annals Phys. 144 (1982) 249 [INSPIRE].

[40] A. Ishibashi and R.M. Wald, Dynamics in nonglobally hyperbolic static space-times. 3. Anti-de Sitter space-time, Class. Quant. Grav. 21 (2004) 2981 [hep-th/0402184] [INSPIRE].

[41] F.G. Friedlander, The wave equation on a curved space-time, Cambridge Monographs on Mathematical Physics, Cambridge University Press, Cambridge U.K. (1975).

[42] S. Hollands and R.M. Wald, Local Wick polynomials and time ordered products of quantum fields in curved space-time, Commun. Math. Phys. 223 (2001) 289 [gr-qc/0103074] [INSPIRE].

[43] S. Hollands and R.M. Wald, On the renormalization group in curved space-time, Commun. Math. Phys. 237 (2003) 123 [gr-qc/0209029] [INSPIRE].

[44] S. Hollands and R.M. Wald, Conservation of the stress tensor in interacting quantum field theory in curved spacetimes, Rev. Math. Phys. 17 (2005) 227 [gr-qc/0404074] [InSPIRE].

[45] R. Brunetti, K. Fredenhagen and M. Kohler, The Microlocal spectrum condition and Wick polynomials of free fields on curved space-times, Commun. Math. Phys. 180 (1996) 633 [gr-qc/9510056] [INSPIRE].

[46] C.P. Burgess and C.A. Lütken, Propagators and Effective Potentials in Anti-de Sitter Space, Phys. Lett. B 153 (1985) 137 [inSPIRE].

[47] R.F. Streater and A.S. Wightman, PCT, spin and statistics, and all that, Advanced book classics. Addison-Wesley, Redwood City, U.S.A. (1989). 
[48] A. Strohmaier, R. Verch and M. Wollenberg, Microlocal analysis of quantum fields on curved space-times: Analytic wavefront sets and Reeh-Schlieder theorems, J. Math. Phys. 43 (2002) 5514 [math-ph/0202003] [INSPIRE].

[49] I. Heemskerk, D. Marolf, J. Polchinski and J. Sully, Bulk and Transhorizon Measurements in AdS/CFT, JHEP 10 (2012) 165 [arXiv:1201.3664] [INSPIRE].

[50] H. Casini, M. Huerta and R.C. Myers, Towards a derivation of holographic entanglement entropy, JHEP 05 (2011) 036 [arXiv:1102.0440] [INSPIRE].

[51] W.G. Unruh and R.M. Wald, What happens when an accelerating observer detects a Rindler particle, Phys. Rev. D 29 (1984) 1047 [InSPIRE].

[52] N.D. Birrell and P.C.W. Davies, Quantum fields in curved space. Cambridge University Press, Cambridge U.K. (1982).

[53] S. Åminneborg, I. Bengtsson, D. Brill, S. Holst and P. Peldán, Black holes and wormholes in $2+1$ dimensions, Class. Quant. Grav. 15 (1998) 627.

[54] K. Krasnov, Holography and Riemann surfaces, Adv. Theor. Math. Phys. 4 (2000) 929 [hep-th/0005106] [INSPIRE].

[55] K. Krasnov, Black hole thermodynamics and Riemann surfaces, Class. Quant. Grav. 20 (2003) 2235 [gr-qc/0302073] [INSPIRE].

[56] K. Skenderis and B.C. van Rees, Holography and wormholes in 2+1 dimensions, Commun. Math. Phys. 301 (2011) 583 [arXiv:0912.2090] [INSPIRE].

[57] S.H. Shenker and D. Stanford, Multiple Shocks, arXiv:1312.3296 [InSPIRE].

[58] K. Papadodimas and S. Raju, An Infalling Observer in AdS/CFT, JHEP 10 (2013) 212 [arXiv: 1211.6767] [INSPIRE].

[59] E. Verlinde and H. Verlinde, Behind the Horizon in AdS/CFT, arXiv:1311.1137 [INSPIRE].

[60] S.G. Avery and B.D. Chowdhury, No Holography for Eternal AdS Black Holes, arXiv:1312.3346 [INSPIRE].

[61] V. Balasubramanian, B. Czech, K. Larjo and J. Simon, Integrability versus information loss: A Simple example, JHEP 11 (2006) 001 [hep-th/0602263] [INSPIRE].

[62] V. Balasubramanian, D. Marolf and M. Rozali, Information Recovery From Black Holes, Gen. Rel. Grav. 38 (2006) 1529 [hep-th/0604045] [INSPIRE].

[63] D. Marolf, Unitarity and Holography in Gravitational Physics, Phys. Rev. D 79 (2009) 044010 [arXiv: 0808.2842] [inSPIRE].

[64] D. Marolf, Holographic Thought Experiments, Phys. Rev. D 79 (2009) 024029 [arXiv: 0808.2845] [INSPIRE].

[65] D. Marolf, Holography without strings?, Class. Quant. Grav. 31 (2014) 015008 [arXiv: 1308.1977] [INSPIRE].

[66] L. Hörmander, The Analysis of Linear Partial Differential Operators I: Distribution Theory and Fourier Analysis, Springer-Verlag, Berlin/Heidelberg, 2nd ed. (1990).

[67] M.J. Radzikowski, Micro-local approach to the Hadamard condition in quantum field theory on curved space-time, Commun. Math. Phys. 179 (1996) 529 [InSPIRE].

[68] R. Verch, Wavefront sets in algebraic quantum field theory, Commun. Math. Phys. 205 (1999) 337 [math-ph/9807022] [INSPIRE]. 